\title{
Power Splitting and Source-Relay Selection in Energy Harvesting Wireless Network
}

xiao jiang ( $\sim 2020040224 @ n j u p t . e d u . c n$ )

Nanjing University of Posts and Telecommunications https://orcid.org/0000-0002-3953-2683

Peng Li

Nanjing University of Posts and Telecommunications

ruchuan wang

Nanjing University of Posts and Telecommunications

\section{Research Article}

Keywords: Energy harvesting, power splitting, source-relay selection, outage probability

Posted Date: May 28th, 2021

DOl: https://doi.org/10.21203/rs.3.rs-335081/v1

License: (1) This work is licensed under a Creative Commons Attribution 4.0 International License. Read Full License

Version of Record: A version of this preprint was published at Wireless Personal Communications on January 4th, 2022. See the published version at https://doi.org/10.1007/s11277-021-09449-1. 


\title{
Power Splitting and Source-Relay Selection in Energy Harvesting Wireless Network
}

\author{
Xiao Jiang, Peng Li and Ruchuan Wang
}

\begin{abstract}
In this paper, we consider an energy-harvesting (EH) relay network composing of multiple sources, a destination and multiple EH decode-and-forward (DF) relays. The EH relays all equip with a power splitter to divide the received signal power into two parts, one for information decoding and the remaining for signal relaying. The power splitting ratio (PSR) depicts the trade-off between the relaying energy and decoding energy. We propose an optimal power splitting and joint sourcerelay selection (OPS-JSRS) scheme where the optimal powersplitting ratio is obtained and the best source-relay pair is selected to transmit the message. For the purpose of comparison, we examine the optimal power splitting and round-robin (OPS-RR) scheme and the traditional power splitting and joint sourcerelay selection (TPS-JSRS) scheme. The exact and asymptotic closed-form expressions of outage probability for OPS-RR, TPSJSRS and OPS-JSRS schemes are derived. Numerical results show that OPS-JSRS scheme is better than OPS-RR and TPSJSRS schemes in terms of outage probability, explaining the superiority of the proposed OPS-JSRS scheme. Additionally, outage probability performance of OPS-JSRS scheme can be improved by increasing the number of sources and relays.
\end{abstract}

Index Terms-Energy harvesting, power splitting, source-relay selection, outage probability.

\section{INTRODUCTION}

$\mathbf{R}$ Ecently, wireless cooperative communications have emerged as a promising technology to reduce channel fading loss and improve the transmission reliability [1]-[5]. Typically, relaying protocols can be divided into two types, namely decode-and-forward (DF) and amplify-and-forward (AF) [6]. Specifically, in the AF protocol, the relay simply amplifies and retransmits the received signals to destination, which suffers from noise propagation problems. By contrast, in the DF protocol, the relay decodes source messages from the received signals first and forwards the decoded messages to destination. In the past few years, the best relay selection has been drawing an increasing attention in the cooperative communications [7]-[9]. Furthermore, the multiple relay selection scheme is proposed to improve the security and reliability of the relay network [10], [11].

The subject is sponsored by the National Key R\&D Program of China (No. 2018YFB1003201), the National Natural Science Foundation of P. R. China (No. 61672296, No. 61872196, No. 61872194, and No. 61902196), Scientific and Technological Support Project of Jiangsu Province (No. BE2017166, and No. BE2019740), Major Natural Science Research Projects in Colleges and Universities of Jiangsu Province (No. 18KJA520008), Six Talent Peaks Project of Jiangsu Province (RJFW-111).(Corresponding author:Peng Li.)

$\mathrm{X}$. Jiang, $\mathrm{P}$. $\mathrm{Li}$ and $\mathrm{R}$. Wang are with the School of Computer Science, Nanjing University of Posts and Telecommunications, Nanjing 210023, China, and also with Jiangsu High Technology Research Key Laboratory for Wireless Sensor Networks, Jiangsu Province, Nanjing 210023, China (E-mail:2020040224@njupt.edu.cn; lipeng@njupt.edu.cn; wangrc@njupt.edu.cn).
Energy harvesting $(\mathrm{EH})$, which can extract energy from surrounding environment, is emerging as an efficient technology to extend the lifetime of the wireless devices, and has been widely used in the wireless communications [12]-[14]. Unlike traditional EH technology, simultaneous wireless information and power transfer (SWIPT) transmits both the information and energy to destination [15]. SWIPT has two architectures, they are time-switching (TS) architecture and power-splitting (PS) architecture [16]. PS architecture means that the received signal power is divided into two parts according to the powersplitting ratio, one for energy harvesting and the remaining for information processing. By contrast, TS architecture indicates that the time slot is divided into two components. In the first time slot, the devices harvest energy from RF signals and the harvested energy is used for information transmission in the second time slot [17].

As a further development, the combination of $\mathrm{EH}$ and cooperative communications has regarded as a hot topic [18][20]. In [18], the authors presented an EH relay system consisting of a source, a destination and multiple EH relays with the existence of eavesdroppers. The relay selection schemes based on DF and AF were proposed to ensure the maximum of security capacity. [19] presented an energy aware relay selection scheme in the EH cognitive radio network for enhancing the security and reliability of the system. In [20], the authors proposed a joint relay-and-antenna selection scheme to minimize the system outage probability in the EH MIMO relay network.

Additionally, there is also some literature devoted to investigating power allocation for EH relay networks [21]-[24]. [21] proposed an optimal power allocation scheme for EH cooperative wireless network and derived the closed-form solutions. In [22], the authors formulated a power allocation problem by maximizing the energy efficiency. The closedform solution is obtained with the aid of nonlinear fractional programming. Additionally, [23] provided a power allocation scheme for maximizing the energy efficiency by obtaining the optimal transmission power of a source in terms of consumed energy fraction. In [24], the authors presented an energy harvesting relay network consisting of a source, an energyharvesting relay and a destination, the source and destination transmit the confidential and interference information to relay, respectively. The optimal power allocation between the source and the destination has been obtained to achieve the maximum secrecy rate.

Presently, only a few studies have focused on the joint power allocation and relay selection for EH relay networks [25], [26]. In [25], the authors formulated an outage probability 


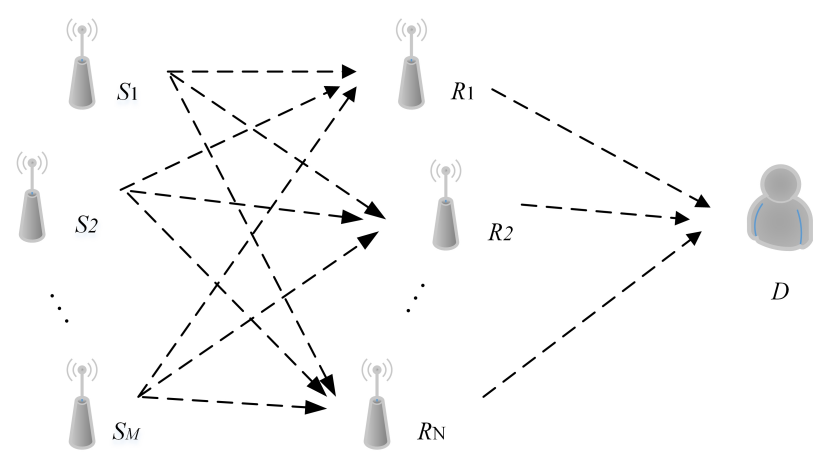

Fig. 1. An energy harvesting relay system consisting of multi-source, one destination and multiple energy-harvesting relays.

minimization problem under the constraints of total transmit power for a two-way AF relay network. [26] investigated a relay selection scheme for EH AF relay networks and solved the joint optimization of relay selection and power splitting. Differing from [25] and [26], where the AF protocol was adopted, in this paper, we jointly consider the power splitting and source-relay selection to improve the outage probability performance in an EH DF relay network.

The main contributions of this paper can be summarized as follows. Firstly, we propose an optimal power splitting and joint source-relay selection (OPS-JSRS) scheme for an EH DF relay network, which differs from [25] and [26], where the AF protocol was used. This is also different from [18] and [19], which only considered the relay selection and did not optimize the power splitting. The power splitting ratio, which refers to the ratio of the harvested energy to total received energy of the relay, depicts the trade-off between the harvesting energy and decoding energy. In the proposed OPS-JSRS scheme, we derive the expression of optimal power-splitting ratio. Additionally, the optimal power splitting and round robin (OPSRR) and traditional power splitting and joint source-relay selection (TPS-JSRS) schemes are proposed for comparison. Secondly, we derive the exact and asymptotic closed-form outage probability expressions of OPS-JSRS, OPS-RR and TPS-JSRS schemes. The numerical results show that the OPSJSRS scheme outperforms OPS-RR and TPS-JSRS schemes in terms of outage probability.

The rest of this paper is organized as follows. Section II presents the system model of a multi-source multi-relay network. In Section III, we propose the OPS-JSRS scheme. For the purpose of comparison, the OPS-RR and TPS-JSRS schemes are introduced. Then the exact closed-form outage probability expressions of OPS-JSRS, OPS-RR and TPS-JSRS schemes are derived. And in Section IV, we obtain the asymptotic closed-form outage probability expressions of OPS-JSRS, OPS-RR and TPS-JSRS schemes. Next, we give the numerical results of the three schemes in Section V. Finally, we conclude the paper in Section VI.

\section{System MODEL}

Fig. 1 presents a multi-source multi-relay system consisting of $M$ sources, represent by $S_{m}, m \in\{1,2, \ldots, M\}$,

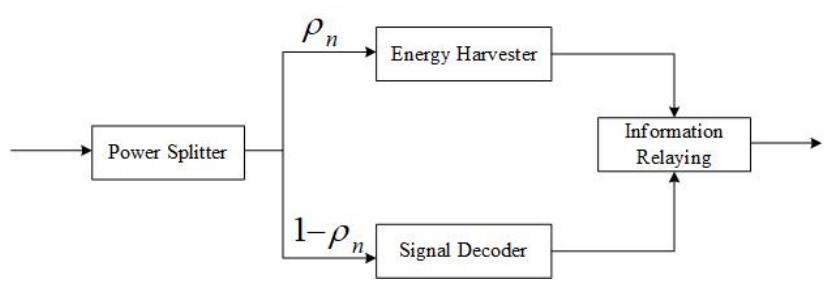

Fig. 2. A block diagram of an EH relay.

a destination $D$ and $N$ EH relays, denote by $R_{n}$, $n \in\{1,2, \ldots, N\}$. Assuming that all nodes have only one antenna and there is no direct link between $S_{m}$ and $D$. All relays equip with a power splitter. Fig. 2 shows a block diagram of an EH relay $R_{n}$, where power splitter divides the energy of $R_{n}$ received from source signal into two parts, which are respectively send to energy harvester of $R_{n}$ for information forwarding and to signal decoder for information processing.

Without loss of generality, let $P$ denote the transmit power of source $S_{m}$. The power-splitting ratio (PSR) of EH relay $R_{n}$ is represented by $\rho_{n}\left(0 \leq \rho_{n} \leq 1\right)$, which refers to the ratio of the harvested energy to total received energy of the relay $R_{n}$. Under Rayleigh fading model, let $h_{S m R n}, h_{R n D}$ denote the fading coefficient of the channel spanning from $S_{m}$ to $R_{n}$ and $R_{n}$ to $D$, respectively. The $\left|h_{S m R n}\right|^{2}$ and $\left|h_{R n D}\right|^{2}$ obey the exponentially distribution with respective means of $\sigma_{S m R n}^{2}$ and $\sigma_{R n D}^{2}$. Therefore, the probability distribution functions (PDFs) of $\left|h_{S m R n}\right|^{2}$ and $\left|h_{R n D}\right|^{2}$ can be expressed as

$$
f_{|h S m R n|^{2}}(x)=\frac{1}{\sigma_{S m R n}^{2}} \exp \left(-\frac{x}{\sigma_{S m R n}^{2}}\right),
$$

and

$$
f_{\left|h_{R n D}\right|^{2}}(x)=\frac{1}{\sigma_{R n D}^{2}} \exp \left(-\frac{x}{\sigma_{R n D}^{2}}\right),
$$

In the first time slot $T$, a source is selected to broadcast $x_{s}\left(E\left[\left|x_{s}\right|^{2}\right]=1\right)$ with a transmit power of $P$ to the $N$ relays, each relay $R_{n}$ divides the received signal power into two components according to the PSR $\rho_{n}$. Without loss of generality, we consider the source $S_{m}$ is selected. Therefore, the signal received at the $R_{n}$ can be obtained as

$$
y_{S m R n}=h_{S m R n} \sqrt{P} x_{s}+n_{S m R n},
$$

where $n_{S m R n}$ is the additive white Gaussian noise (AWGN) at $R_{n}$ and its variance is $N_{0}$. As aforementioned, the relay $R_{S m R n}$ divides the total energy received from source signal $x_{s}$ into two parts. The fraction $\rho_{n}$ of the total energy received from source signal $x_{s}$ is collected by energy harvester for information relaying, and the remaining fraction $1-\rho_{n}$ is used for information decoding. Therefore, we can express the harvested energy at the relay $R_{n}$ in the first slot $T$ as

$$
E_{n}=\rho_{n} \eta P\left|h_{S m R n}\right|^{2} T,
$$

where $\eta$ is a energy conversion efficiency of the energy harvester. Hence, the transmission power used for information relaying during the following time slot of the relay $R_{n}$ can be expressed as

$$
P_{n}^{t}=\rho_{n} \eta P\left|h_{S m R n}\right|^{2}
$$


As mentioned above, the relay $R_{n}$ uses the remaining fraction $1-\rho_{n}$ of received total energy to process the information. Therefore, the power for information processing of $R_{n}$ can be obtained as

$$
P_{n}^{d}=\left(1-\rho_{n}\right) P\left|h_{S m R n}\right|^{2} .
$$

The channel capacity between $S_{m}$ and $R_{n}$ can be expressed as

$$
C_{S m R n}=\frac{1}{2} \log \left(1+\left(1-\rho_{n}\right) \gamma\left|h_{S m R n}\right|^{2}\right),
$$

where $\gamma=\frac{P}{N_{0}}$.

In the second time slot $T$, an EH relay is selected to forward information. Without loss of generality, we consider the EH relay $R_{n}$ is selected to forward the information with the power $P_{n}^{t}$ to the destination $D$, the signal received at $D$ can be written as

$$
y_{R n D}=h_{R n D} \sqrt{\rho_{n} \eta P\left|h_{S m R n}\right|^{2}} x_{s}+n_{R n D},
$$

where $n_{R n D}$ is AWGN at $D$ with variance $N_{0}$. The channel capacity from $R_{n}$ to $D$ can be given by

$$
C_{R n D}=\frac{1}{2} \log \left(1+\rho_{n} \gamma \eta\left|h_{S m R n}\right|^{2}\left|h_{R n D}\right|^{2}\right) .
$$

It has been shown in [1], an overall channel capacity from $S_{m}$ to $D$ via a DF relay is the minimum of the channel capacity from $S_{m}$ to relay $R_{n}$ and that from relay $R_{n}$ to $D$. Hence, we can obtain the overall channel capacity from $S_{m}$ via an EH relay $R_{n}$ to $D$ as

$$
C_{S m R n D}=\min \left(C_{S m R n}, C_{R n D}\right) \text {. }
$$

\section{Power Splitting And Joint Source-Relay SELECTION}

In this section, we propose OPS-JSRS scheme in which the optimal PSR of the relay $R_{n}$ is obtained by maximizing the overall channel capacity from $S_{m}$ to $D$ and a best sourcerelay pair is chosen. In order to compare the proposed power splitting scheme, we examine the TPS-JSRS in which the PSR is not optimized. We also propose OPS-RR scheme to verify the superiority of joint source-relay selection scheme. Exact closed-form outage probability expressions of the OPS-RR, OPS-JSRS and TPS-JSRS schemes are derived over Rayleigh fading channels.

\section{A. TPS-JSRS Scheme}

In this subsection, we present the TPS-JSRS scheme and derive its closed-form outage probability expression. In TPSJSRS scheme, the PSR $\rho_{n}$ is not optimized and varies in the interval of $[0,1]$. Meanwhile, we select the best source-relay pair by maximizing the overall channel capacity from $S_{m}$ to $D$. Accordingly, the best source-relay pair selection criterion can be expressed as

$$
\left(S_{m^{*}}, R_{n^{*}}\right)=\arg \max _{\substack{1 \leq m \leq M \\ 1 \leq n \leq N}} C_{S m R n D},
$$

where $C_{S m R n D}$ is given by (10).

Following, the outage probability of the TPS-JSRS scheme is analyzed. An outage event occurs when the overall channel capacity drops below a predefined date rate $R[10]$. The outage probability of the TPS-JSRS scheme can be expressed as (12) at the top of the following page, where $\beta=\left(2^{2 R}-1\right) / \gamma$, and $\left|h_{S m^{*} R_{n}}\right|^{2}=\max _{1 \leq m \leq M}\left|h_{S m R n}\right|^{2}$.

Notice that $\left|h_{S m R n}\right|^{2}$ follows exponential distributions with the mean of $\sigma_{S m R n}^{2}$. Thus, the cumulative density function (CDF) of $\left|h_{S m^{*} R_{n}}\right|^{2}$ can be expressed as

$$
F_{\left|h_{S m^{*} R_{n}}\right|^{2}}(x)=\prod_{m=1}^{M}\left[1-\exp \left(-\frac{x}{\sigma_{S m R n}^{2}}\right)\right] .
$$

Therefore, the PDF of $\left|h_{S m * R n}\right|^{2}$ can be obtained as

$$
\begin{aligned}
f_{\left|h_{S m^{*} R_{n}}\right|^{2}}(x) & =\sum_{i=1}^{M} \frac{1}{\sigma_{S i R n}^{2}} \exp \left(-\frac{x}{\sigma_{S i R n}^{2}}\right) \\
& \times \prod_{m=1, m \neq i}^{M}\left[1-\exp \left(-\frac{x}{\sigma_{S m R n}^{2}}\right)\right] .
\end{aligned}
$$

Denoting $X=\left|h_{S m^{*} R_{n}}\right|^{2}$,the $P_{\text {out }, 1}^{\mathrm{TPS}-\mathrm{JSRS}}$ can be rewritten as

$$
\begin{aligned}
P_{\text {out }, 1}^{\mathrm{TPS}-J S R S} & =\operatorname{Pr}\left(X>a,\left|h_{R n D}\right|^{2}>\frac{\beta}{\rho_{n} \eta X}\right) \\
& =\int_{a}^{\infty} \exp \left(-\frac{\beta}{\sigma_{R n D}^{2} \rho_{n} \eta x}\right) \sum_{i=1}^{M} \frac{1}{\sigma_{S i R n}^{2}} \exp \left(-\frac{x}{\sigma_{\text {SiRn }}^{2}}\right) \\
& \times \prod_{m=1, m \neq i}^{M}\left[1-\exp \left(-\frac{x}{\sigma_{\text {SmRn }}^{2}}\right)\right] d x
\end{aligned}
$$

where $a=\frac{2^{2 R}-1}{\left(1-\rho_{n}\right) \gamma}$. Using the binomial formula, we have

$$
\begin{aligned}
& \prod_{m=1, m \neq i}^{M}\left[1-\exp \left(-\frac{x}{\sigma_{S m R n}^{2}}\right)\right] \\
= & 1+\sum_{k=1}^{2^{M-1}-1}(-1)^{\left|D_{k}\right|} \exp \left(-\sum_{S m \in D_{k}} \frac{x}{\sigma_{S m R n}^{2}}\right)
\end{aligned}
$$

where $D_{k}$ represents the k-th nonempty subset of $M$ sources, $\left|D_{k}\right|$ represents the number of elements in set $D_{k}$. Thus, the $P_{\text {out }, 1}^{\text {TPS-JSRS }}$ can be rewritten as

$$
P_{\text {out }, 1}^{\mathrm{TPS}-\mathrm{JSRS}}=\Phi_{1}+\Phi_{2}
$$

where

$\Phi_{1}=\sum_{i=1}^{M} \int_{a}^{\infty} \frac{1}{\sigma_{\text {SiRn }}^{2}} \exp \left(-\frac{x}{\sigma_{\text {SiRn }}^{2}}\right) \exp \left(-\frac{\beta}{\sigma_{R n D}^{2} \rho_{n} \eta x}\right) d x$

and

$$
\begin{aligned}
\Phi_{2} & =\sum_{i=1}^{M} \frac{1}{\sigma_{S i R n}^{2}} \int_{a}^{\infty} \sum_{k=1}^{2^{M-1}-1}(-1)^{\left|D_{k}\right|} \exp \left(-\sum_{S m \in D_{k}} \frac{x}{\sigma_{S m R n}^{2}}\right) \\
& \times \exp \left(-\frac{x}{\sigma_{\text {SiRn }}^{2}}\right) \exp \left(-\frac{\beta}{\sigma_{R n D}^{2} \rho_{n} \eta x}\right) d x
\end{aligned}
$$

Using the Maclaurin series expansion, we have

$$
\exp \left(-\frac{\beta}{\sigma_{R n D}^{2} \rho_{n} \eta x}\right)=\sum_{u=0}^{\infty} \frac{(-1)^{u} \beta^{u}}{u ! \sigma_{R n D}^{2 u} \rho_{n}^{u} \eta^{u} x^{u}} .
$$

Substituting (20) into (18),we can obtain the $\Phi_{1}$ as (21) at the top of following page, where $\operatorname{Ei}(x)=\int_{x}^{\infty} \frac{e^{t}}{t} d t$ and 


$$
\begin{aligned}
& P_{\text {out }}^{\mathrm{TPS}-\mathrm{JSRS}}=\operatorname{Pr}\left(\max _{\substack{1 \leq m \leq M \\
1 \leq n \leq N}} C_{S m R n D}<R\right)=\operatorname{Pr}\left\{\max _{\substack{1 \leq m \leq M \\
1 \leq n \leq N}}\left[\min \left(\left(1-\rho_{n}\right)\left|h_{S m R n}\right|^{2}, \rho_{n} \eta\left|h_{S m R n}\right|^{2}\left|h_{R n D}\right|^{2}\right)\right]<\beta\right\} \\
& =\prod_{n=1}^{N} \operatorname{Pr}\left\{\min \left(\left(1-\rho_{n}\right)\left|h_{S m * R n}\right|^{2}, \rho_{n} \eta\left|h_{S m * R n}\right|^{2}\left|h_{R n D}\right|^{2}\right)<\beta\right\} \\
& =\prod_{n=1}^{N}[1-\underbrace{\operatorname{Pr}\left(\left(1-\rho_{n}\right)\left|h_{S m * R n}\right|^{2}>\beta, \rho_{n} \eta\left|h_{S m * R n}\right|^{2}\left|h_{R n D}\right|^{2}>\beta\right)}_{P_{\text {out }, 1}^{\text {TPS }- \text { JSRS }}}]
\end{aligned}
$$

$$
\begin{aligned}
\Phi_{1} & =\sum_{i=1}^{M} \frac{1}{\sigma_{S i R n}^{2}} \int_{a}^{\infty} \exp \left(-\frac{x}{\sigma_{S i R n}^{2}}\right) d x-\sum_{i=1}^{M} \frac{\beta}{\sigma_{S i R n}^{2} \sigma_{R n D}^{2} \rho_{n} \eta} \int_{a}^{\infty} \frac{1}{x} \exp \left(-\frac{x}{\sigma_{S i R n}^{2}}\right) d x+\sum_{i=1}^{M} \frac{1}{\sigma_{S i R n}^{2}} \sum_{u=2}^{\infty} \frac{(-1)^{u} \beta^{u}}{u ! \sigma_{R n D}^{2 u} \rho_{n}^{u} \eta^{u}} \Phi_{1, u} \\
& =\sum_{i=1}^{M} \exp \left(-\frac{a}{\sigma_{S i R n}^{2}}\right)-\sum_{i=1}^{M} \frac{\beta}{\sigma_{S i R n}^{2} \sigma_{R n D}^{2} \rho_{n} \eta} \operatorname{Ei}\left(\frac{a}{\sigma_{S i R n}^{2}}\right)+\sum_{i=1}^{M} \frac{1}{\sigma_{S i R n}^{2}} \sum_{u=2}^{\infty} \frac{(-1)^{u} \beta^{u}}{u ! \sigma_{R n D}^{2 u} \rho_{n}^{u} \eta^{u}} \Phi_{1, u}
\end{aligned}
$$

$$
\begin{aligned}
\Phi_{1, u} & =\int_{a}^{\infty} \frac{1}{x^{u}} \exp \left(-\frac{x}{\sigma_{\text {SiRn }}^{2}}\right) d x \\
& =\frac{1}{a^{u-1}} \sum_{k=0}^{u-2} \frac{(-1)^{k} a^{k}}{\sigma_{\text {SiRn }}^{2 k}(u-1)(u-2) \cdots(u-k+1)} \exp \left(-\frac{a}{\sigma_{\text {SiRn }}^{2}}\right) . \\
& +(-1)^{u} \frac{1}{(u-1) ! \sigma_{\text {SiRn }}^{2(u-1)}} \operatorname{Ei}\left(-\frac{a}{\sigma_{\text {SiRn }}^{2}}\right)
\end{aligned}
$$

Similarly, substituting (20) into (19), we can obtain the $\Phi_{2}$ as

$$
\begin{aligned}
\Phi_{2}=\sum_{i=1}^{M} & \sum_{k=1}^{2^{M-1}-1} \frac{(-1)^{\mid D_{k}} \mid}{b \sigma_{\text {SiRn }}^{2}} \exp (-a b) \\
& -\sum_{i=1}^{M} \sum_{k=1}^{2^{M-1}-1} \frac{(-1)^{\left|D_{k}\right|} \beta E i(a b)}{\rho_{n} \eta \sigma_{R n D}^{2} \sigma_{S i R n}^{2}} \\
& +\sum_{i=1}^{M} \sum_{k=1}^{2^{M-1}} \sum_{u=2}^{\infty} \frac{(-1)^{\left|D_{k}\right|+u} \beta^{u}}{\sigma_{S i R n}^{2} u ! \sigma_{R n D}^{2 u} \rho_{n} u \eta^{u}} \Phi_{2, u}
\end{aligned}
$$

where $b=\frac{1}{\sigma_{S i R n}^{2}}+\sum_{S m \in D_{k}} \frac{1}{\sigma_{S m R n}^{2}}$ and

$$
\begin{aligned}
\Phi_{2, u} & =\int_{a}^{\infty} \frac{1}{x^{u}} \exp (-b x) d x \\
& =\frac{1}{a^{u-1}} \sum_{k=0}^{u-2} \frac{(-1)^{k} a^{k} b^{k}}{(u-1)(u-2) \cdots(u-k+1)} \exp (-a b) . \\
& +(-1)^{u} \frac{b^{u-1}}{(u-1) !} \operatorname{Ei}(-a b)
\end{aligned}
$$

Substituting (21) and (23) into (17), we can obtain the closed-form expression of $P_{\text {out }, 1}^{\mathrm{TPS}-\mathrm{JSRS}}$ as

$$
\begin{aligned}
P_{\text {out }, 1}^{\mathrm{TPS}-J S R S} & =\sum_{i=1}^{M} \exp \left(-\frac{a}{\sigma_{S i R n}^{2}}\right)-\sum_{i=1}^{M} \frac{\beta E i\left(\frac{a}{\sigma_{\text {SiRn }}^{2}}\right)}{\sigma_{S i R n}^{2} \sigma_{R n D}^{2} \rho_{n} \eta} \\
& +\sum_{i=1}^{M} \sum_{u=2}^{\infty} \frac{(-1)^{u} \beta^{u}}{u ! \sigma_{R n D}^{2 u} \rho_{n}{ }^{u} \eta^{u}} \Phi_{1, u} \\
& +\sum_{i=1}^{M} \sum_{k=1}^{2^{M-1}-1} \frac{(-1)^{\left|D_{k}\right|}}{b \sigma_{S i R n}^{2}} \exp (-a b) \\
& -\sum_{i=1}^{M} \sum_{k=1}^{2^{M-1}-1} \frac{(-1)^{\left|D_{k}\right|} \beta}{\rho_{n} \eta \sigma_{R n D}^{2} \sigma_{S i R n}^{2}} E i(a b) \\
& +\sum_{i=1}^{M} \sum_{k=1}^{2} \sum_{u=2}^{\infty} \frac{(-1)^{\left|D_{k}\right|+u} \beta^{u}}{\sigma_{S i R n}^{2} u ! \sigma_{R n D}^{2 u} \rho_{n}{ }^{u} \eta^{u}} \Phi_{2, u}
\end{aligned}
$$

where $\Phi_{1, u}$ and $\Phi_{2, u}$ are given by (22) and (24), respectively. Substituting $P_{\text {out }, 1}^{\text {TPS -JSRS }}$ from (25) into (12), the outage probability of TPS - JSRS $P_{\text {out }}^{\text {TPS-JSRS }}$ can be obtained.

\section{B. OPS-RR Scheme}

This subsection presents an optimal power splitting and round-robin (OPS-RR) scheme and derive the closed-form outage probability expression of OPS-RR scheme over Rayleigh fading channels. As mentioned above, the relay $R_{n}$ divides the total energy of received source signal $x_{s}$ into two parts. The fraction $\rho_{n}$ of the received total energy is collected by energy harvester for information forwarding during the following time slot, and the remaining fraction $1-\rho_{n}$ of the total energy is used for information decoding. In this scheme, the optimal PSR $\rho_{n}$ of the relay $R_{n}$ is obtained by maximizing the channel capacity from $S_{m}$ to $D$. This differs from the TPS-JSRS scheme, which does not optimize the PSR $\rho_{n}$. Therefore, the optimal PSR $\rho_{n}$ can be obtained as

$$
\rho_{n}^{*}=\max _{0 \leq \rho_{n} \leq 1} C_{S m R n D}=\max _{0 \leq \rho_{n} \leq 1} \min \left(C_{S m R n}, C_{R n D}\right)
$$

where $C_{S m R n}$ and $C_{R n D}$ are given by (7) and (9), respectively. Combining (7), (9) and (26) yields

$$
\rho_{n}^{*}=\arg \max _{0 \leq \rho_{n} \leq 1} \min \left(1-\rho_{n}, \rho_{n} \eta\left|h_{R n D}\right|^{2}\right) .
$$

We can obtain the optimal PSR $\rho_{n}$ when $1-\rho_{n}$ equals $\rho_{n} \eta\left|h_{n d}\right|^{2}$ as

$$
\rho_{n}^{*}=\frac{1}{1+\eta\left|h_{R n D}\right|^{2}} .
$$

Substituting $\rho_{n}^{*}$ from (28) into (10), the overall channel capacity from $S_{m}$ to $D$ via $\mathrm{EH}$ relay $R_{n}$ can be rewritten as

$$
C_{S m R n D}^{*}=\frac{1}{2} \log \left(1+\frac{\gamma \eta\left|h_{S m R n}\right|^{2}\left|h_{R n D}\right|^{2}}{1+\eta\left|h_{R n D}\right|^{2}}\right) .
$$

Following, a source-relay pair is chosen to transmit the message. In the OPS-RR scheme, each source and the relay is given the equal chance to transmit information. To be specific, 
$M \times N$ different source-relay pairs comprised of $M$ sources and $N$ relays take turns in accessing the channel. Without loss of generality, the source $S_{m}$ and the relay $R_{n}$ are chosen. Therefore, the outage probability of OPS-RR scheme from $S_{m}$ to $D$ via the relay $R_{n}$ can be expressed as

$$
P_{\text {out }, \text { SmRn }}^{\text {OPS-RR }}=\operatorname{Pr}\left(C_{S m R n D}^{*}<R\right) .
$$

Substituting $C_{S m R n D}^{*}$ from (29) into (30) gives

$$
P_{\text {out }, \text { SmRn }}^{\text {OPS-RR }}=\operatorname{Pr}\left(\frac{1}{2} \log \left(1+\frac{\gamma \eta\left|h_{S m R n}\right|^{2}\left|h_{R n D}\right|^{2}}{1+\eta\left|h_{R n D}\right|^{2}}\right)<R\right),
$$

which is further simplified to

$$
P_{\text {out }, S m R n}^{\text {OPS-RR }}=\operatorname{Pr}\left(\left|h_{S m R n}\right|^{2}<\frac{\alpha}{\left|h_{R n D}\right|^{2}}+\alpha \eta\right),
$$

where $\alpha=\frac{2^{2 R}-1}{\gamma \eta}$. Notice that $\left|h_{S m R n}\right|^{2}$ and $\left|h_{R n D}\right|^{2}$ follow exponential distributions with means of $\sigma_{S m R n}^{2}$ and $\sigma_{R n D}^{2}$. Denoting $X=\left|h_{S m R n}\right|^{2}$ and $Y=\left|h_{R n D}\right|^{2}$, the $P_{\text {out }, S m R n}^{O P S R R}$ can be rewritten as (33) at the top of the following page, where $K_{1}(\cdot)$ is the first-order modified Bessel function of second kind as given by (8.432.6) in [29].

As mentioned above, the OPS-RR scheme gives each source and relay the equal chance to be selected for information transmission. Therefore, the outage probability of OPS-RR scheme is the mean of $M \times N$ source-relay pairs' outage probability, yields

$$
P_{\text {out }}^{\mathrm{OPS}-\mathrm{RR}}=\sum_{m=1}^{M} \sum_{n=1}^{N} \frac{1}{M \times N} P_{\text {out }, \text { SmRn }}^{\mathrm{OPS}} .
$$

where the $P_{\text {out }, S m R n}^{\mathrm{OPS}-\mathrm{RR}}$ is given by (33).

\section{OPS-JSRS Scheme}

This subsection proposes an optimal power splitting and joint source-relay selection (OPS-JSRS) scheme. And the closed-form outage probability expression of OPS-JSRS scheme is derived. In OPS-JSRS scheme, the optimal PSR $\rho_{n}$ is obtained by maximizing the overall channel capacity from $S_{m}$ to $D$ via an EH relay $R_{n}$, which is same as OPSRR scheme. Therefore, the optimal PSR of OPS-JSRS scheme is equal to that of OPS-RR scheme and is given by (28). Furthermore, the overall channel capacity $C_{S m R n D}^{*}$ from $S_{m}$ to $D$ via EH relay $R_{n}$ of OPS-JSRS scheme is given by (29).

In the OPS-JSRS scheme, a source-relay pair having the maximal overall channel capacity of $C_{S m R n D}^{*}$ is chosen to transmit message. Therefore, the source-relay pair selection criterion for the OPS-JSRS scheme can be defined as

$$
\begin{aligned}
\left(S_{m^{*}}, R_{n^{*}}\right)=\arg \max _{\substack{1 \leq m \leq M \\
1 \leq n \leq N}} C_{S m R n D}^{*} \\
=\arg \max _{\substack{1 \leq m \leq M \\
1 \leq n \leq N}} \frac{\left|h_{S m R n}\right|^{2}\left|h_{R n D}\right|^{2}}{1+\eta\left|h_{R n D}\right|^{2}}
\end{aligned}
$$

Therefore, the overall channel capacity of OPS-JSRS scheme can be expressed as

$$
C^{\text {OPS-JSRS }}=\max _{\substack{1 \leq m \leq M \\ 1 \leq n \leq N}} C_{S m R n D}^{*},
$$

where $C_{S m R n D}^{*}$ is given by (29).

The following present an outage probability analysis of OPS-JSRS scheme. As mentioned above, an outage event occurs when the overall channel capacity $C^{\text {OPS-JSRS }}$ drops below a predefined data rate $R$. Hence, we can obtain the outage probability of the OPS-JSRS scheme as

$$
\begin{aligned}
P_{\text {out }}^{\text {OPS-JSRS }} & =\operatorname{Pr}\left(C^{\text {OPS-JSRS }}<R\right) \\
& =\operatorname{Pr}\left(\max _{\substack{1 \leq m \leq M \\
1 \leq n \leq N}} C_{S m R n D}^{*}<R\right),
\end{aligned}
$$

where $C_{S m R n D}^{*}$ is given by (29). Substituting $C_{S m R n D}^{*}$ from (29) into (37), the $P_{\text {out }}^{\text {OPS-JSRS }}$ can be rewritten as

$$
\begin{gathered}
P_{\text {out }}^{\text {OPS-JSRS }}=\operatorname{Pr}\left[\max _{\substack{1 \leq m \leq M \\
1 \leq n \leq N}} \frac{1}{2} \log \left(1+\frac{\gamma \eta\left|h_{S m R n}\right|^{2}\left|h_{R n D}\right|^{2}}{1+\eta\left|h_{R n D}\right|^{2}}\right)<R\right] \\
=\prod_{n=1}^{N} \operatorname{Pr}\left[\max _{1 \leq m \leq M} \frac{1}{2} \log \left(1+\frac{\gamma \eta\left|h_{S m R n}\right|^{2}\left|h_{R n D}\right|^{2}}{1+\eta\left|h_{R n D}\right|^{2}}\right)<R\right] \\
=\prod_{n=1}^{N} \operatorname{Pr}\left(\left|h_{S m^{*} R_{n}}\right|^{2}<\frac{\alpha}{\left|h_{R n D}\right|^{2}}+\alpha \eta\right)
\end{gathered}
$$

where $\left|h_{S m^{*} R_{n}}\right|^{2}=\max _{1 \leq m \leq M}\left|h_{S m R n}\right|^{2}$. Notice that $\left|h_{S m R n}\right|^{2}$ follows exponential distributions with mean of $\sigma_{S m R n}^{2}$. Thus, CDF of $\left|h_{S m^{*} R_{n}}\right|^{2}$ can be expressed as

$$
F_{\left|h_{S m^{*} R_{n}}\right|^{2}}(x)=\prod_{m=1}^{M}\left[1-\exp \left(-\frac{x}{\sigma_{S m R n}^{2}}\right)\right]
$$

Denoting $Y=\left|h_{R n D}\right|^{2}$, the $P_{\text {out }}^{\text {OPS-JSRS }}$ can be rewritten as

$$
\begin{aligned}
P_{\text {out }}^{\text {OPS-JSRS }}= & \prod_{n=1}^{N} \int_{0}^{\infty} f_{Y}(y) F_{\left|h_{S m^{*} R n}\right|^{2}}\left(\frac{\alpha}{y}+\alpha \eta\right) d y \\
= & \prod_{n=1}^{N} \int_{0}^{\infty} \frac{1}{\sigma_{R n D}^{2}} \exp \left(-\frac{y}{\sigma_{R n D}^{2}}\right) \\
& \times \prod_{m=1}^{M}\left[1-\exp \left(-\frac{\alpha}{\sigma_{S m R n}^{2} y}-\frac{\alpha \eta}{\sigma_{S m R n}^{2}}\right)\right] d y
\end{aligned}
$$

Using the binomial formula, we have

$$
\begin{aligned}
& \prod_{m=1}^{M}\left[1-\exp \left(-\frac{\alpha}{\sigma_{S m R n}^{2} y}-\frac{\alpha \eta}{\sigma_{S m R n}^{2}}\right)\right] \\
& =1+\sum_{j=1}^{2^{M}-1}(-1)^{\left|A_{j}\right|} \exp \left(-\sum_{S m \in A_{j}}\left(\frac{\alpha}{\sigma_{S m R n}^{2} y}+\frac{\alpha \eta}{\sigma_{S m R n}^{2}}\right)\right)
\end{aligned}
$$

where $A_{j}$ represents the $\mathrm{j}$-th nonempty subset of $M$ sources, $\left|A_{j}\right|$ represents the number of elements in set $A_{j}$. Substituting (41) into (40), we can obtain the outage probability of OPSJSRS scheme $P_{\text {out }}^{\text {OPS-JSRS }}$ as (42) at the top of the following page.

\section{Asymptotic Outage Probability Analysis}

To obtain more insights, in this section we analyze the asymptotic outage probability in the high SNR region with $\gamma \rightarrow \infty$ 


$$
\begin{aligned}
P_{\text {out }, S m R n}^{\text {OPS }-\mathrm{RR}} & =\int_{0}^{\infty} f_{Y}(y) F_{X}\left(\frac{\alpha}{y}+\alpha \eta\right) d y=\int_{0}^{\infty} \frac{1}{\sigma_{R n D}^{2}} \exp \left(-\frac{y}{\sigma_{R n D}^{2}}\right)\left[1-\exp \left(-\frac{\alpha}{\sigma_{S m R n}^{2} y}-\frac{\alpha \eta}{\sigma_{S m R n}^{2}}\right)\right] d y \\
& =1-\exp \left(-\frac{\alpha \eta}{\sigma_{S m R n}^{2}}\right) \int_{0}^{\infty} \frac{1}{\sigma_{R n D}^{2}} \exp \left(-\frac{y}{\sigma_{R n D}^{2}}-\frac{\alpha}{\sigma_{S m R n}^{2} y}\right) d y \\
& =1-\exp \left(-\frac{\alpha \eta}{\sigma_{S m R n}^{2}}\right) \frac{2 \sqrt{\alpha}}{\sigma_{R n D} \sigma_{S m R n}} K_{1}\left(\frac{2 \sqrt{\alpha}}{\sigma_{R n D} \sigma_{S m R n}}\right)
\end{aligned}
$$

$$
\begin{aligned}
& P_{\text {out }}^{\text {OPS-JSRS }}=\prod_{n=1}^{N} \int_{0}^{\infty} \frac{1}{\sigma_{R n D}^{2}} \exp \left(-\frac{y}{\sigma_{R n D}^{2}}\right)\left[1+\sum_{j=1}^{2^{M}-1}(-1)^{\left|A_{j}\right|} \exp \left(-\sum_{S m \in A_{j}}\left(\frac{\alpha}{\sigma_{S m R n}^{2} y}+\frac{\alpha \eta}{\sigma_{S m R n}^{2}}\right)\right)\right] d y \\
& =\prod_{n=1}^{N}\left[1+\sum_{j=1}^{2^{M}-1}(-1)^{\left|A_{j}\right|} \exp \left(-\sum_{S m \in A_{j}} \frac{\alpha \eta}{\sigma_{S m R n}^{2}}\right) \int_{0}^{\infty} \frac{1}{\sigma_{R n D}^{2}} \exp \left(-\frac{y}{\sigma_{R n D}^{2}}-\sum_{S m \in A_{j}} \frac{\alpha}{\sigma_{S m R n}^{2} y}\right) d y\right] \\
& =\prod_{n=1}^{N}\left[1+\sum_{j=1}^{2^{M}-1}(-1)^{\left|A_{j}\right|} \exp \left(-\sum_{S m \in A_{j}} \frac{\alpha \eta}{\sigma_{S m R n}^{2}}\right) \frac{2}{\sigma_{R n D}} \sqrt{\sum_{S m \in A_{j}} \frac{\alpha}{\sigma_{S m R n}^{2}}} K_{1}\left(\frac{2}{\sigma_{R n D}} \sqrt{\sum_{S m \in A_{j}} \frac{\alpha}{\sigma_{S m R n}^{2}}}\right)\right]
\end{aligned}
$$

\section{A. TPS-JSRS Scheme}

In the high SNR region, the asymptotic outage probability of TPS-JSRS scheme can be expressed as

$$
P_{\text {out }}^{T P S-J S R S, \infty}=\left(1-\Phi_{1}^{\infty}-\Phi_{2}^{\infty}\right)^{N}
$$

where

$$
\begin{aligned}
\Phi_{1}^{\infty} & =\sum_{i=1}^{M} \int_{0}^{\infty} \frac{1}{\sigma_{S i R n}^{2}} \exp \left(-\frac{x}{\sigma_{S i R n}^{2}}\right) \exp \left(-\frac{\beta}{\sigma_{R n D}^{2} \rho_{n} \eta x}\right) d x \\
& =\sum_{i=1}^{M} 2 \sqrt{\frac{\beta}{\sigma_{S i R n}^{2} \sigma_{R n D}^{2} \rho_{n} \eta}} K_{1}\left(2 \sqrt{\frac{\beta}{\sigma_{S i R n}^{2} \sigma_{R n D}^{2} \rho_{n} \eta}}\right)
\end{aligned}
$$

and

$$
\begin{aligned}
\Phi_{2}^{\infty}= & \sum_{i=1}^{M} \frac{1}{\sigma_{S i R n}^{2}} \int_{0}^{\infty} \sum_{k=1}^{2^{M-1}-1}(-1)^{\left|D_{k}\right|} \\
\times & \exp \left(-\sum_{S m \in D_{k}} \frac{x}{\sigma_{S m R n}^{2}}-\frac{x}{\sigma_{S i R n}^{2}}-\frac{\beta}{\sigma_{R n D}^{2} \rho_{n} \eta x}\right) d x \\
= & \sum_{i=1}^{M} \frac{1}{2^{M-1}-1} \sum_{k=1}^{2 i R n}(-1)^{\left|D_{k}\right|} \\
& \times 2 \sqrt{\frac{\beta}{\sigma_{R n D}^{2} \rho_{n} \eta b}} K_{1}\left(2 \sqrt{\frac{\beta b}{\sigma_{R n D}^{2} \rho_{n} \eta}}\right)
\end{aligned}
$$

Substituting (44) and (45) into (43), the asymptotic outage probability of the TPS-JSRS scheme can be obtained.

\section{B. OPS-RR Scheme}

When $\gamma \rightarrow \infty$, the asymptotic outage probability of OPSRR scheme can be obtained as

$$
P_{\text {out }}^{\text {OPS-RR, } \infty}=\sum_{m=1}^{M} \sum_{n=1}^{N} \frac{1}{M \times N} \operatorname{Pr}\left(\left|h_{S m R n}\right|^{2}<\frac{\alpha}{\left|h_{R n D}\right|^{2}}\right) \text {. }
$$

Similar to the derivation of (33), (46) can be obtained as (47).

$$
\begin{aligned}
P_{\text {out }}^{\text {OPS }-\mathrm{RR}, \infty} & =\sum_{m=1}^{M} \sum_{n=1}^{N} \frac{1}{M \times N} \int_{0}^{\infty} \frac{1}{\sigma_{R n D}^{2}} \exp \left(-\frac{y}{\sigma_{R n D}^{2}}\right) \\
& \times\left[1-\exp \left(-\frac{\alpha}{\sigma_{S m R n}^{2} y}\right)\right] d y \\
= & \sum_{m=1}^{M} \sum_{n=1}^{N} \frac{1}{M \times N} \\
- & \sum_{m=1}^{M} \sum_{n=1}^{N} \frac{1}{M \times N} \frac{2 \sqrt{\alpha}}{\sigma_{R n D} \sigma_{S m R n}} K_{1}\left(\frac{2 \sqrt{\alpha}}{\sigma_{R n D} \sigma_{S m R n}}\right)
\end{aligned}
$$

\section{OPS-JSRS Scheme}

In the high SNR region, the asymptotic outage probability of OPS-JSRS scheme can be obtained as

$$
P_{\text {out }}^{\text {OPS-JSRS, } \infty}=\prod_{n=1}^{N} \operatorname{Pr}\left(\left|h_{S m^{*} R_{n}}\right|^{2}<\frac{\alpha}{\left|h_{R n D}\right|^{2}}\right) .
$$

Similar to (42), the $P_{\text {out }}^{\text {OPS-JSRS, } \infty}$ can be derived as

$$
\begin{aligned}
& P_{\text {out }}^{\text {OPS-JSRS }, \infty}=\prod_{n=1}^{N} \int_{0}^{\infty} \frac{1}{\sigma_{R n D}^{2}} \exp \left(-\frac{y}{\sigma_{R n D}^{2}}\right) \\
& \times\left[1+\sum_{j=1}^{2^{M}-1}(-1)^{\left|A_{j}\right|} \exp \left(-\sum_{S m \in A_{j}} \frac{\alpha}{\sigma_{S m R n}^{2} y}\right)\right] d y \\
& \quad=\prod_{n=1}^{N}\left[1+\sum_{j=1}^{2^{M}-1}(-1)^{\left|A_{j}\right|}\right. \\
& \left.\times \frac{2}{\sigma_{R n D}} \sqrt{\sum_{S m \in A_{j}} \frac{\alpha}{\sigma_{S m R n}^{2}}} K_{1}\left(\frac{2}{\sigma_{R n D}} \sqrt{\sum_{S m \in A_{j}} \frac{\alpha}{\sigma_{S m R n}^{2}}}\right)\right]
\end{aligned}
$$

\section{NUMERICAL RESUlTS AND Discussions}

In this section, we present the numerical and simulation results for OPS-RR, OPS-JSRS and TPS-JSRS schemes in terms of their outage probability. All the numerical results are based on the above-mentioned analysis. We assume that the transmission links between any two nodes of Fig. 1 are Rayleigh fading channels. Following the existing literature[17]-[19], we assume that all relays have the same power splitting ratio $\rho_{n}=\rho$, and the average channel gains of $\sigma_{S m R n}^{2}=\sigma_{R n D}^{2}=1$, the energy conversion efficiency of $\eta=0.5, \rho_{n}=0.5$ are used.

Fig. 3 shows the theoretical and simulated outage probability versus power splitting ratio $\rho$ of the OPS-JSRS and TPS-JSRS schemes with $\mathrm{SNR}=10 \mathrm{~dB}, R=1 \mathrm{bit} / \mathrm{s} / \mathrm{Hz}, \eta=0.5$ and $M=N=4$. The power splitting ratio $\rho$ of TPS-JSRS scheme is not optimized and varies from 0 to 1 , while in the OPS-JSRS scheme, an optimized $\rho$ is given by (28). As shown in Fig. 3, with the increase of $\rho$, the outage probability curve of TPS-JSRS scheme first decreases and then rises, illustrating that the outage probability of TPS-JSRS scheme can be minimized by optimizing $\rho$. It can also be seen from Fig. 3, outage probability of TPS-JSRS scheme is higher than that of OPS-JSRS scheme, explaining the superiority of the proposed power splitting scheme. 


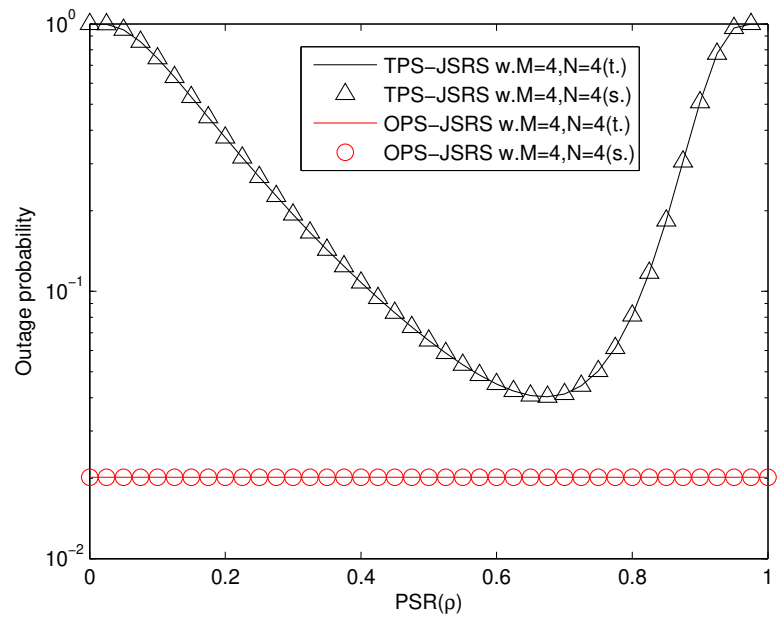

Fig. 3. Outage probability versus PSR $\rho$ of the OPS-JSRS and TPS-JSRS schemes with $\mathrm{SNR}=10 \mathrm{~dB}, R=1 \mathrm{bit} / \mathrm{s} / \mathrm{Hz}, \eta=0.5$ and $M=N=4$, where t. and s. represent the theoretical and simulated outage probability results, respectively.

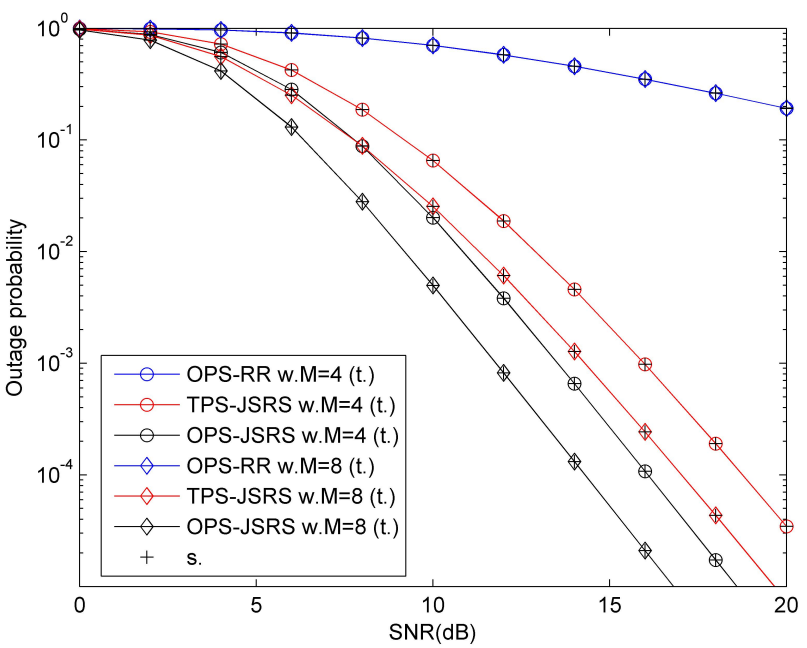

Fig. 4. Outage probability versus SNR of the OPS-JSRS as well as TPS-JSRS and OPS-RR schemes for different number of sources with $R=1 \mathrm{bit} / \mathrm{s} / \mathrm{Hz}$ $\eta=0.5$ and $N=4$, where t. and s. represent the theoretical and simulated outage probability results, respectively.

Fig. 4 illustrates the theoretical and simulated outage probability versus SNR of the OPS-JSRS as well as TPS-JSRS and OPS-RR schemes for different number of sources of $M=4$ and $M=8$ with $R=1 \mathrm{bit} / \mathrm{s} / \mathrm{Hz}, \eta=0.5$ and $N=4$. As shown from Fig. 4, with the SNR increases, outage probabilities of OPS-JSRS as well as TPS-JSRS and OPS-RR schemes decrease, that means the outage probability performance can be improved by increasing the SNR. One can also observe from Fig.4 that the outage probability of OPS-JSRS scheme is lower than that of OPS-RR scheme and TPS-JSRS scheme. Moreover, with the increase of the number of sources from $M=4$ to $M=8$, the outage probability of OPS-JSRS decreases while that of OPS-RR scheme remains unchanged, demonstrating that the OPS-JSRS scheme outperforms OPS-

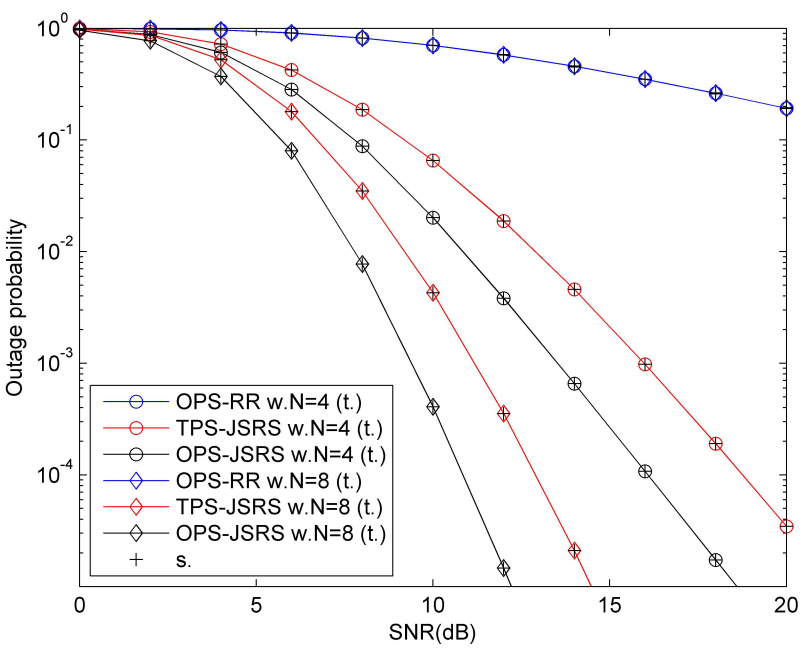

Fig. 5. Outage probability versus SNR of the OPS-JSRS as well as TPS-JSRS and OPS-RR schemes for different number of EH relays with $R=1 \mathrm{bit} / \mathrm{s} / \mathrm{Hz}$, $\eta=0.5$ and $M=4$, where t. and s. represent the theoretical and simulated outage probability results, respectively.

RR scheme in terms of outage probability and the outage advantage becomes more significant as the number of sources increases. Additionally, the outage performance of proposed OPS-JSRS scheme can be improved by increasing the number of sources.

Fig. 5 depicts the theoretical and simulated outage probability versus SNR of the OPS-JSRS as well as TPS-JSRS and OPS-RR schemes for different number of EH relays of $N=4$ and $N=8$, we assume the data rate $R=1 \mathrm{bit} / \mathrm{s} / \mathrm{Hz}$, the energy conversion efficiency $\eta=0.5$ and the number of sources $M=4$. It can be seen from Fig. 5, in the whole SNR region, the outage probability of the proposed OPSJSRS scheme is lower than that of OPS-RR scheme and TPSJSRS scheme. That means the proposed OPS-JSRS scheme is better than OPS-RR scheme and TPS-JSRS scheme in terms of outage probability. It also appears from Fig. 5 that with the increasing of the number of EH relays from $N=4$ to $N=8$, the outage probability of OPS-JSRS scheme decreases, implying that the outage probability performance of proposed OPS-JSRS scheme can be greatly improved by increasing the number of EH relays.

In Fig. 6, we present the theoretical and simulated outage probability versus SNR of OPS-JSRS as well as TPS-JSRS and OPS-RR schemes for different data rates of $R=1 \mathrm{bit} / \mathrm{s} / \mathrm{Hz}$ and $R=0.5 \mathrm{bit} / \mathrm{s} / \mathrm{Hz}$ with $\eta=0.5$ and $M=N=4$. As shown in Fig. 6, with the data rate decreases from $R=1 \mathrm{bit} / \mathrm{s} / \mathrm{Hz}$ to $R=0.5 \mathrm{bit} / \mathrm{s} / \mathrm{Hz}$, outage probabilities of OPS-JSRS, TPSJSRS and OPS-RR schemes decrease. That is to say, outage probability performances of OPS-JSRS, TPS-JSRS and OPSRR schemes can be enhanced by decreasing the data rate. One can also observe from Fig. 6, the OPS-JSRS scheme is better than the OPS-RR scheme and TPS-JSRS scheme in terms of outage probability whatever $R=1 \mathrm{bit} / \mathrm{s} / \mathrm{Hz}$ or $R=0.5 \mathrm{bit} / \mathrm{s} / \mathrm{Hz}$, verifying the superiority of proposed OPSJSRS scheme. 


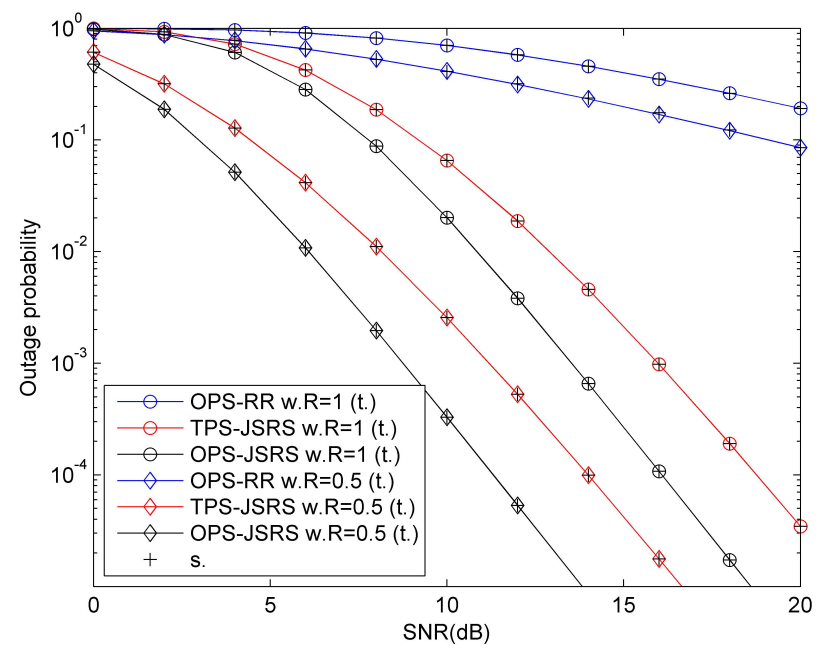

Fig. 6. Outage probability versus SNR of the OPS-JSRS as well as TPS-JSRS and OPS-RR schemes for different data rates with $\eta=0.5$ and $M=N=4$, where $t$. and $s$. represent the theoretical and simulated outage probability results, respectively.

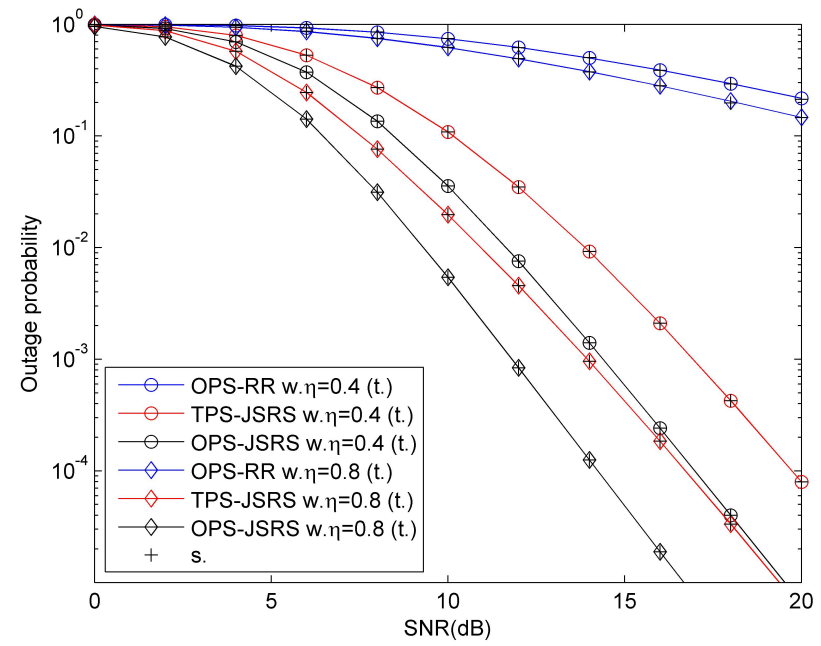

Fig. 7. Outage probability versus SNR of the OPS-JSRS as well as TPSJSRS and OPS-RR schemes for different energy conversion efficiency with $R=1 \mathrm{bit} / \mathrm{s} / \mathrm{Hz}$ and $M=N=4$, where t. and s. represent the theoretical and simulated outage probability results, respectively.

Fig. 7 shows the theoretical and simulated outage probability versus SNR of the OPS-JSRS as well as TPS-JSRS and OPS-RR schemes for different energy conversion efficiency $\eta=0.4$ and $\eta=0.8$ with $R=1 \mathrm{bit} / \mathrm{s} / \mathrm{Hz}$ and $M=N=4$. It can be seen from Fig. 7, as the energy conversion efficiency increases from $\eta=0.4$ to $\eta=0.8$, outage probabilities of OPSJSRS, TPS-JSRS and OPS-RR schemes decrease. Due to the fact that with the increase of $\eta$, the relay converts more energy from received RF signals for information relaying, which leads to a lower outage probability. Fig.7 also demonstrates that the OPS-JSRS scheme outperforms OPS-RR scheme and TPSJSRS scheme in terms of outage probability.

Fig. 8 illustrates the theoretical and simulated outage probability versus energy conversion efficiency $\eta$ of the OPS-JSRS

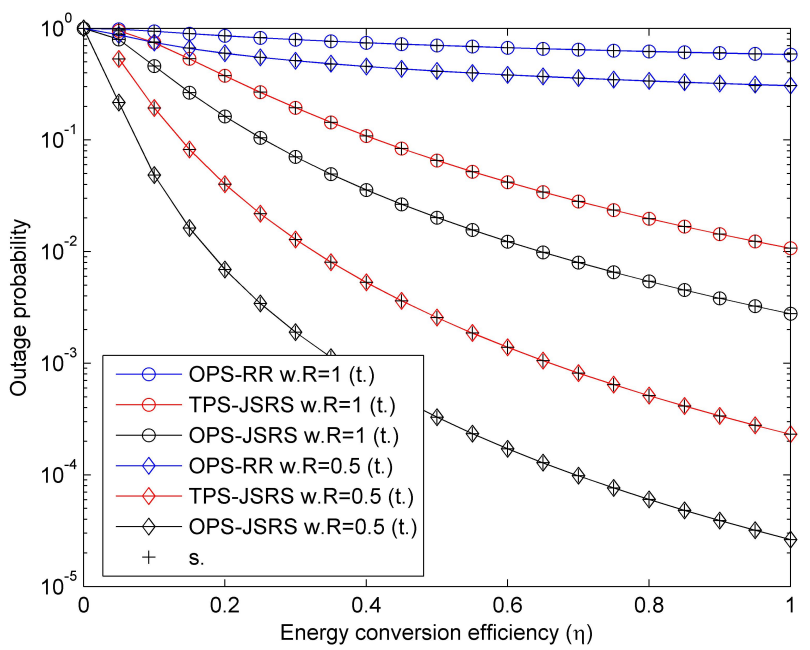

Fig. 8. Outage probability versus energy conversion efficiency $\eta$ of the OPSJSRS as well as TPS-JSRS and OPS-RR schemes for different data rates with $\mathrm{SNR}=10$ and $M=N=4$, where t. and s. represent the theoretical and simulated outage probability results, respectively.

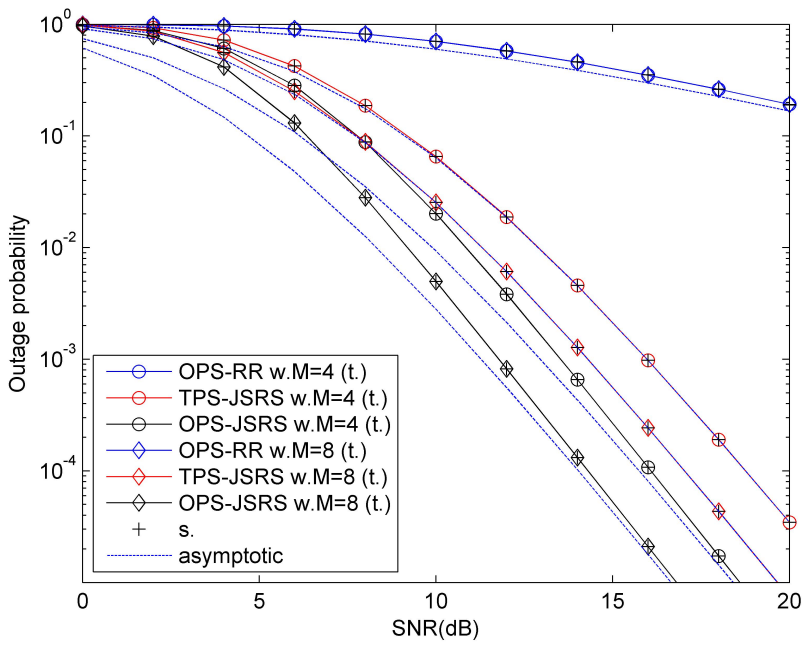

Fig. 9. Exact and asymptotic outage probability versus SNR of the OPSJSRS as well as TPS-JSRS and OPS-RR schemes for different number of sources with $R=1 \mathrm{bit} / \mathrm{s} / \mathrm{Hz}, \eta=0.5$ and $N=4$, where t. and s. represent the theoretical and simulated outage probability results, respectively.

as well as TPS-JSRS and OPS-RR schemes for different data rates of $R=1 \mathrm{bit} / \mathrm{s} / \mathrm{Hz}$ and $R=0.5 \mathrm{bit} / \mathrm{s} / \mathrm{Hz}$ with $\mathrm{SNR}=10$ and $M=N=4$. It can be seen from Fig. 8, as the increase of $\eta$ from 0 to 1 , outage probabilities of OPS-JSRS, TPS-JSRS and OPS-RR schemes decrease, demonstrating that outage probabilities of OPS-JSRS, TPS-JSRS and OPS-RR schemes are affected by $\eta$. In other words, the higher energy conversion efficiency, the better outage probability performance.

Fig. 9 shows the exact and asymptotic outage probability versus SNR of the OPS-JSRS as well as TPS-JSRS and OPSRR schemes with $R=1 \mathrm{bit} / \mathrm{s} / \mathrm{Hz}, \eta=0.5$ and $N=4$. It can be seen from Fig. 9, the asymptotic outage probability curves of OPS-JSRS, TPS-JSRS and OPS-RR schemes match the exact ones very well in the high SNR region. 


\section{CONCLUTIONS}

In this paper, we proposed an optimal power splitting and joint source-relay selection (OPS-JSRS) scheme in energy harvesting DF relay system consisting of $M$ sources, a destination and $N$ EH relays. In OPS-JSRS scheme, we calculated and derived the expression of the optimal power-splitting ratio (PSR) and selected the best source-relay pair to transmit the messages. For the purpose of comparison, we presented the traditional power splitting and joint source-relay scheme (TPS-JSRS) in which the PSR was not optimized and the optimal power splitting and round robin (OPS-RR) scheme. We derived the exact and asymptotic closed-form outage probability expressions of OPS-JSRS, TPS-JSRS and OPS-RR schemes. Numerical results showed that the outage probability of OPS-JSRS scheme is lower than that of TPS-JSRS scheme and OPS-RR scheme, indicating that the proposed OPSJSRS scheme has outage advantage. Additionally, the outage probability performance of OPS-JSRS schemes improves with the increase of the number of sources and relays.

\section{REFERENCES}

[1] Y. Zou, J. Zhu, and R. Zhang, "Exploiting network cooperation in green wireless communication," IEEE Transactions on Communications, vol. 61, no. 3, pp. 999-1010, Mar. 2013.

[2] D. Mishra, S. De, and D. Krishnaswamy, "Dilemma at RF Energy Harvesting Relay: Downlink Energy Relaying or Uplink Information Transfer?," IEEE Transactions on Wireless Communications, vol. 16, no. 8, pp. 4939-4955, Aug. 2017.

[3] Y. Wu, A. Khisti, C. Xiao, G. Caire, K. Wong, and X. Gao, "A Survey of Physical Layer Security Techniques for 5G Wireless Networks and Challenges Ahead," IEEE Journal on Selected Areas in Communications, vol. 36, no. 4, pp. 679-695, Apr. 2018.

[4] P. Yan, J. Yang, M. Liu, J. Sun, and G. Gui,, "Secrecy Outage Analysis of Transmit Antenna Selection Assisted With Wireless Power Beacon," IEEE Transactions on Vehicular Technology, vol. 69, no. 7, pp. 74737482, Jul. 2020.

[5] R. Zhu, T. Li, J. Guo, and Y. Huang, "Relay selection scheme for AF system with partial CSI and optimal stopping theory," Tsinghua Science and Technology, vol. 25, no. 2, pp. 302-312, Apr. 2020.

[6] A. Nosratinia, T. E. Hunter, and A. Hedayat, "Cooperative communication in wireless networks," IEEE Communications Magazine, vol. 42, no. 10, pp.74-80, Oct. 2004.

[7] J. Li, L. J. Cimini, J. Ge, C. Zhang, and H. Feng, "Optimal and Suboptimal Joint Relay and Antenna Selection for Two-Way Amplify-andForward Relaying," IEEE Transactions on Wireless Communications, vol. 15, no. 2, pp. 980-993, Feb. 2016.

[8] D. Qiao, "Effective Capacity of Buffer-Aided Full-Duplex Relay Systems With Selection Relaying,' IEEE Transactions on Communications, vol. 64, no. 1, pp. 117-129, Jan. 2016.

[9] S. Jia, J. Zhang, H. Zhao, Y. Lou, and Y. Xu, "Relay Selection for Improved Physical Layer Security in Cognitive Relay Networks Using Artificial Noise," IEEE Access, vol. 6, pp. 64836-64846, 2018.

[10] J. Zhu, Y. Zou, B. Champagne, W. P. Zhu, and L. Hanzo, "Securityreliability tradeoff analysis of multi relay-aided decode-and-forward cooperation systems," IEEE Transactions on Vehicular Technology, vol. 65, no. 7, pp. 5825-5831, Jul. 2016.

[11] Y. Zou, X. Wang, and W. Shen, "Optimal relay selection for physical layer security in cooperative wireless networks," IEEE Journal on Selected Areas in Communications, vol. 31, no. 10, pp. 2099-2111, Oct. 2013.

[12] P. Yan, Y. Zou, and J. Zhu, "Energy-Aware Multiuser Scheduling for Physical-Layer Security in Energy-Harvesting Underlay Cognitive Radio Systems," IEEE Transactions on Vehicular Technology, vol. 67, no. 3, pp. 2084-2096, Mar. 2018.

[13] J. Ren, J. Hu, D. Zhang, H. Guo, Y. Zhang, and X. Shen, "RF energy harvesting and transfer in cognitive radio sensor networks: Opportunities and challenges," IEEE Communications Magazine,vol. 56, no. 1, pp. 104-110, Jan. 2018.
[14] T. Li, P. Fan, and K. B. Letaief, "Outage probability of energy harvesting relay-aided cooperative networks over Rayleigh fading channel," IEEE Transactions on Vehicular Technology, vol. 65, no. 2, pp. 972-978, Feb. 2016.

[15] R. Zhang and C. K. Ho, "MIMO broadcasting for simultaneous wireless information and power transfer," IEEE Transactions on Wireless Communications, vol. 12, no. 5, pp. 1989-2001, May 2013.

[16] H. Lei, M. Xu, I. S. Ansari, G. Pan, K. A. Qaraqe, and M. Alouini, "On Secure Underlay MIMO Cognitive Radio Networks With Energy Harvesting and Transmit Antenna Selection," IEEE Transactions on Green Communications and Networking, vol. 1, no. 2, pp. 192-203, Jun. 2017.

[17] X. Zhou, R. Zhang, and C. K. Ho, "Wireless information and power transfer: architecture design and rate-energy tradeoff," IEEE Transactions on Communications, vol. 61, no. 11, pp. 4754-4767, Nov. 2013.

[18] P. N. Son and H. Y. Kong, "Cooperative communication with energyharvesting relays under physical layer security," IET Communications, vol. 9, no. 17, pp. 2131-2139, Nov. 2015.

[19] P. Yan, Y. Zou, X. Ding, and J. Zhu, "Energy-Aware Relay Selection Improves Security-Reliability Tradeoff in Energy Harvesting Cooperative Cognitive Radio Systems," IEEE Transactions on Vehicular Technology, vol. 69, no. 5, pp. 5115-5128, May 2020.

[20] J. Men, J. Ge, and C. Zhang, "A joint relay-and-antenna selection scheme in energy-harvesting MIMO relay networks," IEEE Signal Processing Letters, vol. 23, no. 4, pp. 532-536, Apr. 2016.

[21] X. Huang and N. Ansari, "Optimal cooperative power allocation for energy-harvesting-enabled relay networks," IEEE Transactions on Vehicular Technology, vol. 65, no. 4, pp. 2424-2434, Apr. 2016.

[22] C. Zhang, H. Du, and J. Ge, "Energy-Efficient Power Allocation in Energy Harvesting Two-Way AF Relay Systems," IEEE Access, vol. 5, pp. 3640-3645, 2017.

[23] F. K. Ojo, D. O. Akande, and M. F. M. Salleh, "Optimal Power Allocation in Cooperative Networks With Energy-Saving Protocols," IEEE Transactions on Vehicular Technology, vol. 69, no. 5, pp. 50795088, May 2020.

[24] R. Yao, F. Xu, T. Mekkawy, and J. Xu, "Optimised power allocation to maximise secure rate in energy harvesting relay network," Electronics Letters, vol. 52, no. 22, pp. 1879-1881, Oct. 2016.

[25] J. Men, J. Ge, C. Zhang, and J. Li, "Joint optimal power allocation and relay selection scheme in energy harvesting asymmetric two-way relaying system," IET Communications, vol. 9, no. 11, pp. 1421-1426, Jul. 2015 .

[26] A. Alsharoa, H. Ghazzai, A. E. Kamal and A. Kadri, "Optimization ofa power splitting protocol for two-way multiple energy harvesting relay system," IEEE Trans. Green Commun. Net., vol. 1, no. 4, pp. 444-457, Dec. 2017.

[27] L. Elmorshedy and C. Leung, "Power allocation in an RF energy harvesting DF relay network in the presence of an interferer,' IEEE Access, vol. 5, pp. 7606-7618, May. 2017.

[28] Y. Zhou and T.S. Ng, "MIMO-OFCDM systems with joint iterative detection and optimal power allocation," IEEE Transactions on Wireless Communications, vol. 7, no. 12, pp. 5504-5516, Dec. 2008.

[29] I. S. Gradshteyn and I. M. Ryzhik, Tables of Integrals, Series, and Products 7th Ed. New York, NY, USA: Academic Press, 2007.

\section{Data AVAilability}

Not applicable.

\section{Code AVAILABILITy}

The data that support the findings of this study are available from the corresponding author upon request.

\section{CONFLiCts of InTERest}

The authors declare that they have no conflicts of interest. 
Figures

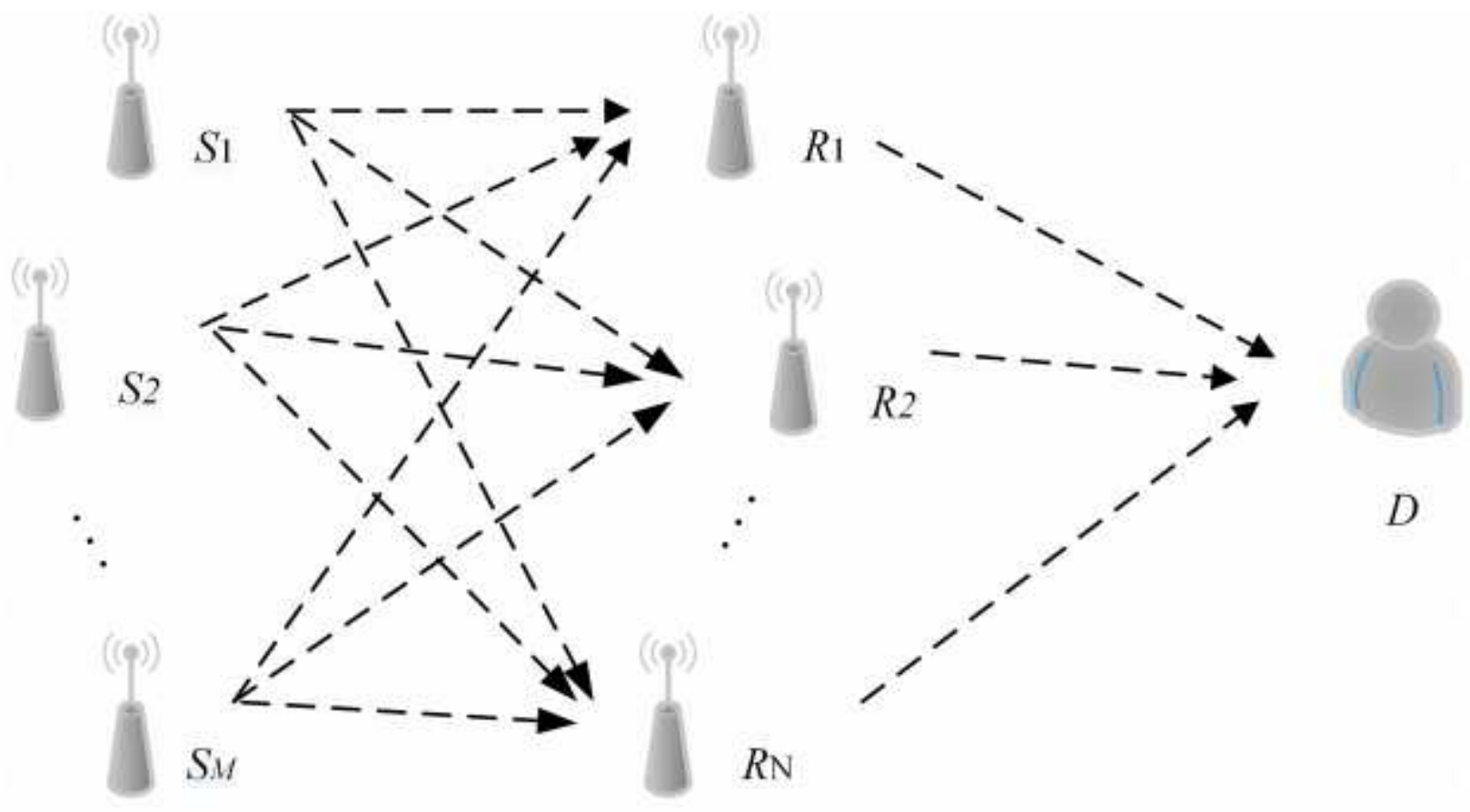

\section{Figure 1}

An energy harvesting relay system consisting of multi-source, one destination and multiple energyharvesting relays.

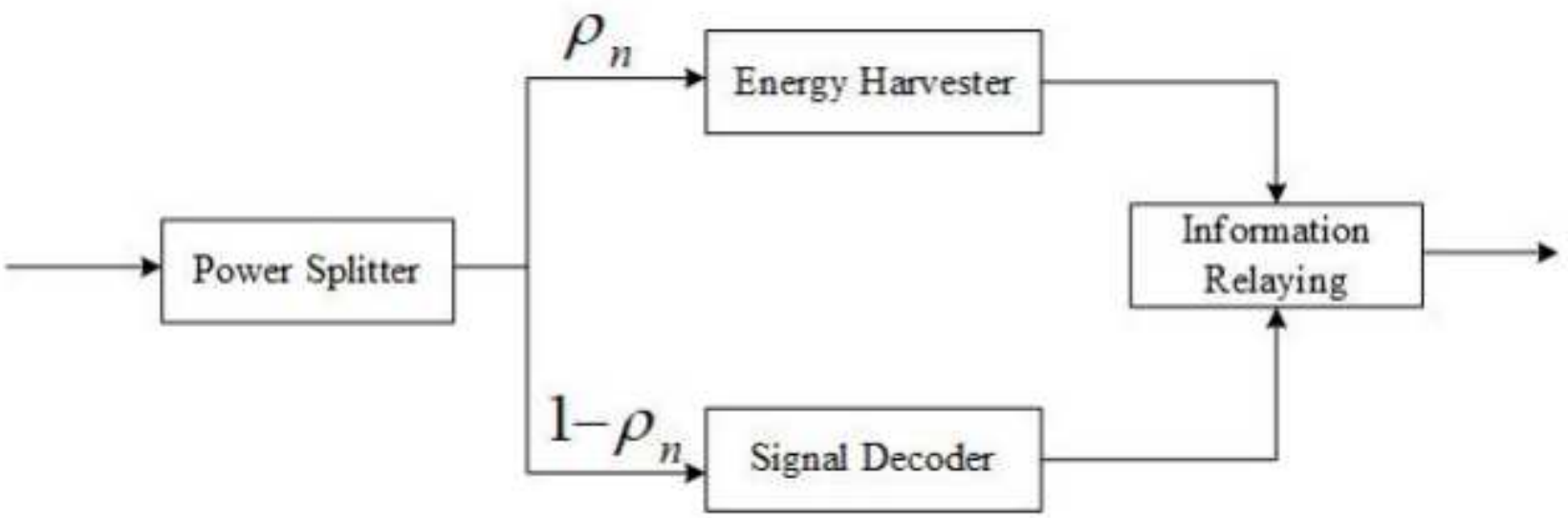

Figure 2

A block diagram of an EH relay. 


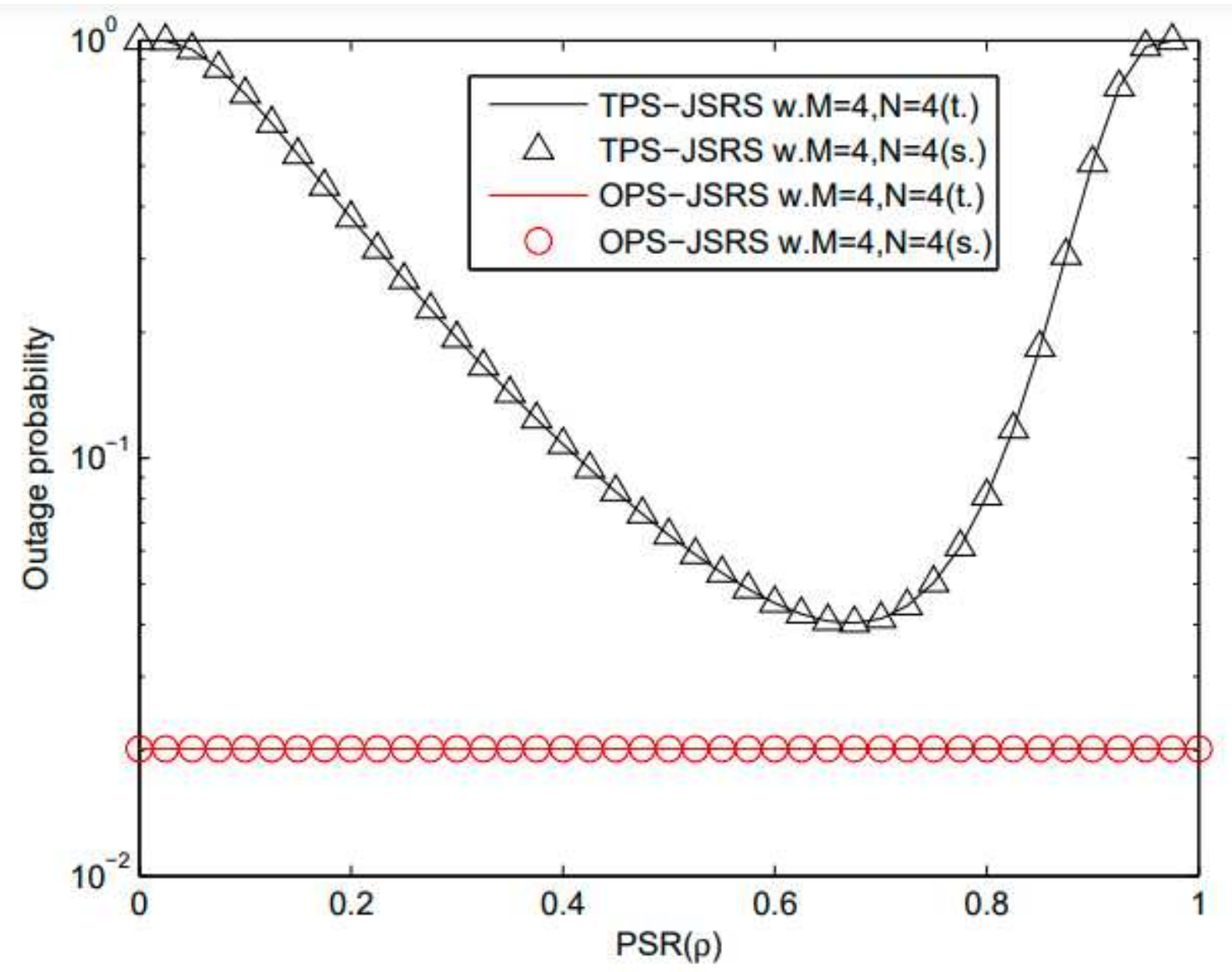

Figure 3

Please see the Manuscript PDF file for the complete figure caption 


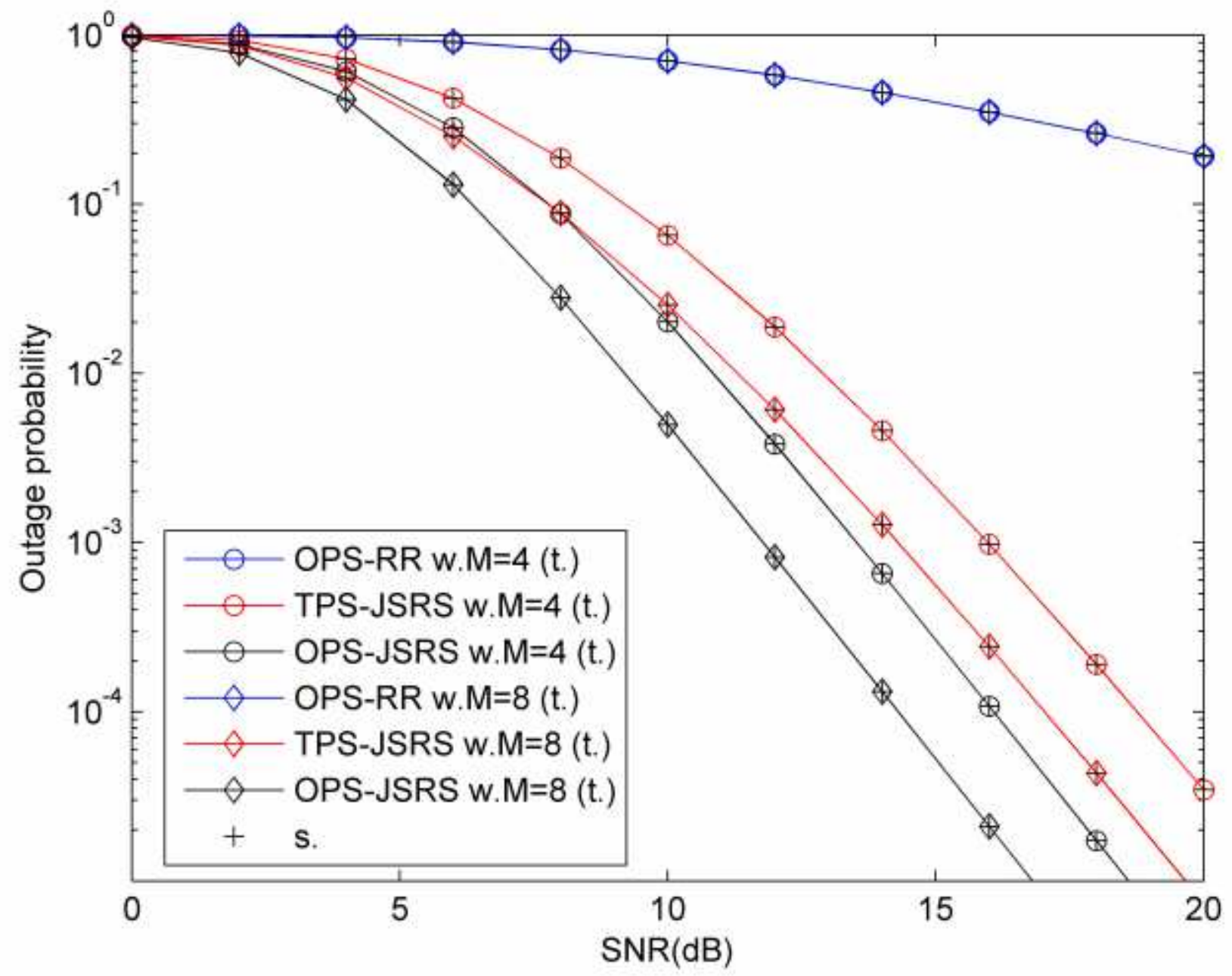

Figure 4

Please see the Manuscript PDF file for the complete figure caption 


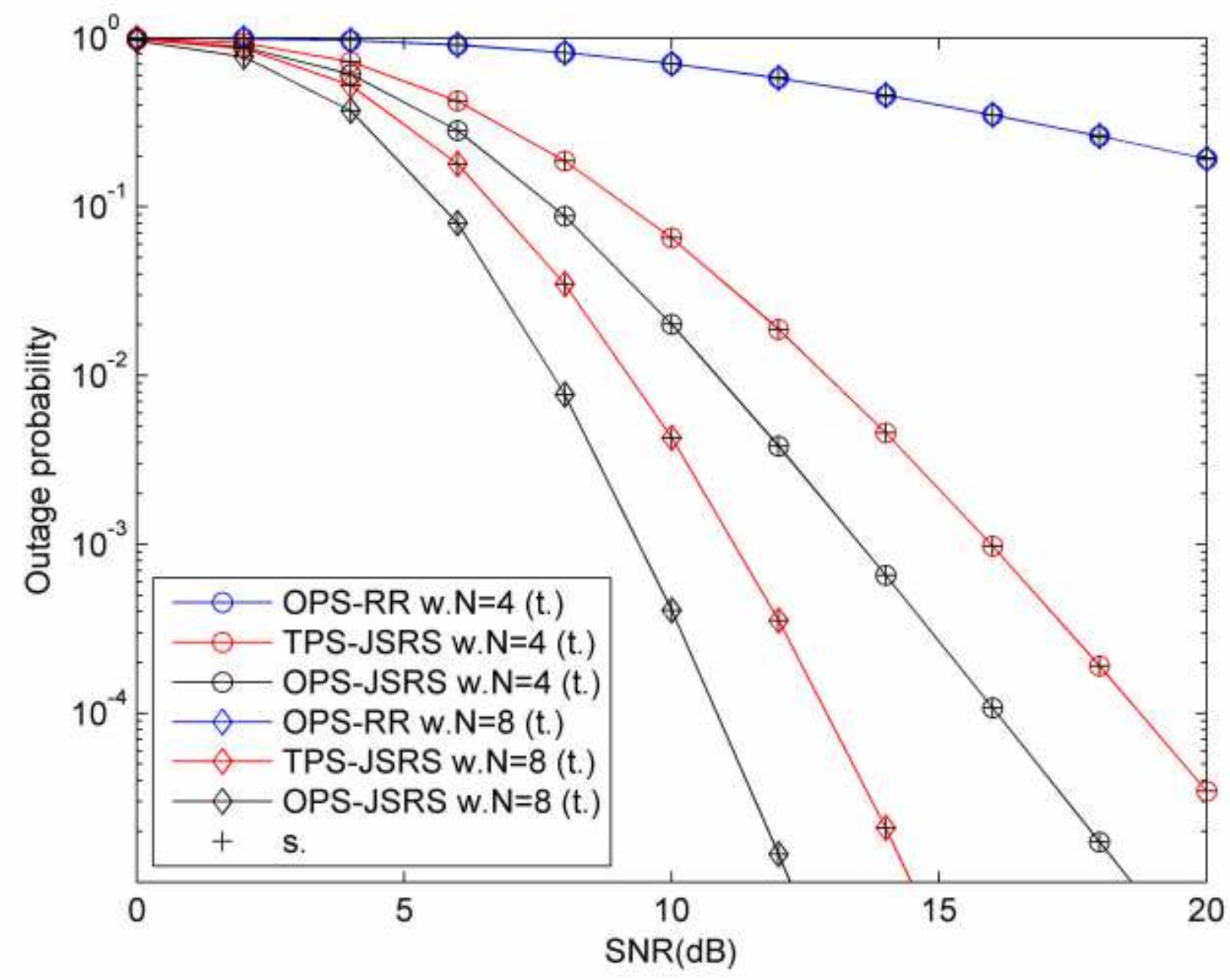

Figure 5

Please see the Manuscript PDF file for the complete figure caption 


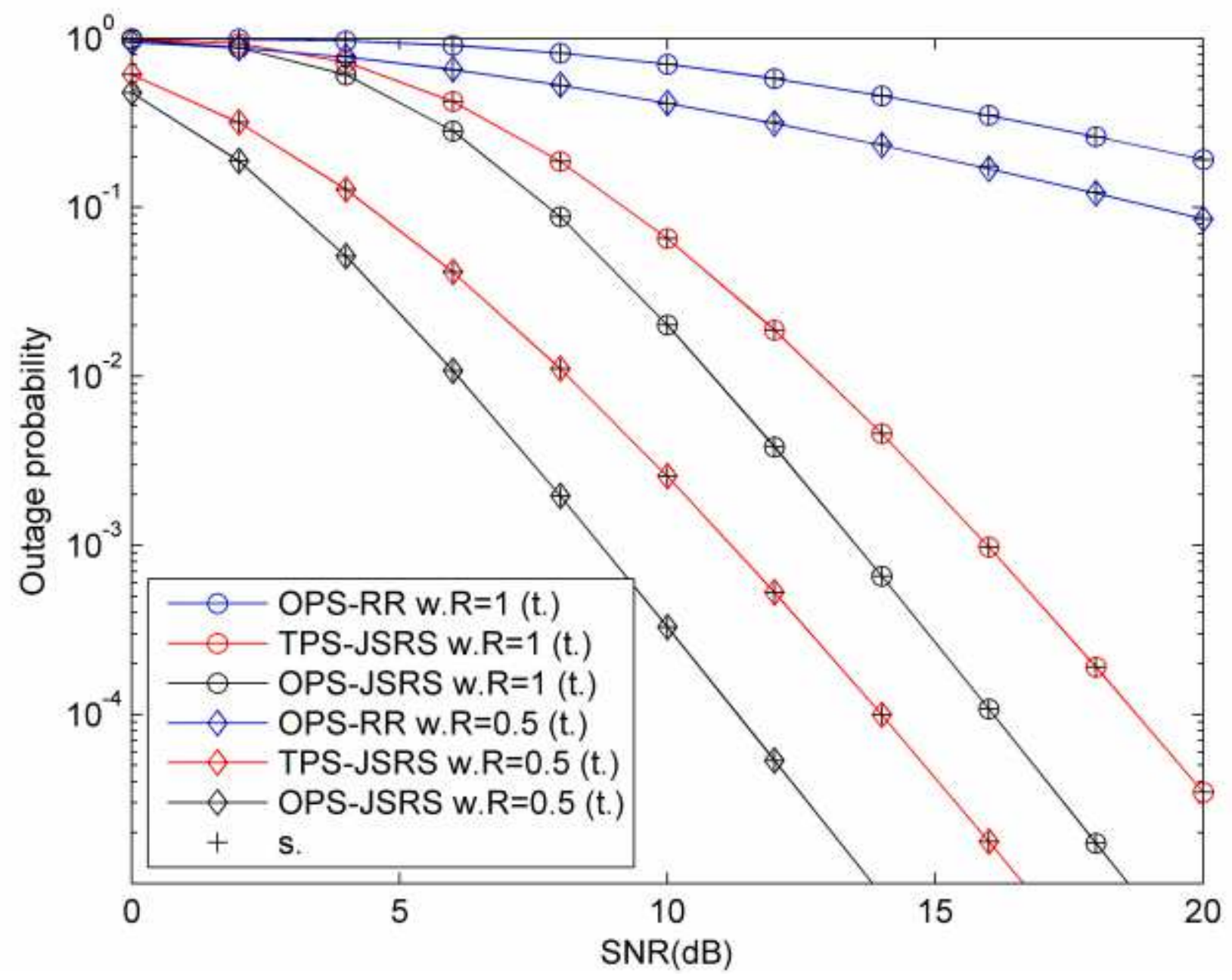

Figure 6

Please see the Manuscript PDF file for the complete figure caption 


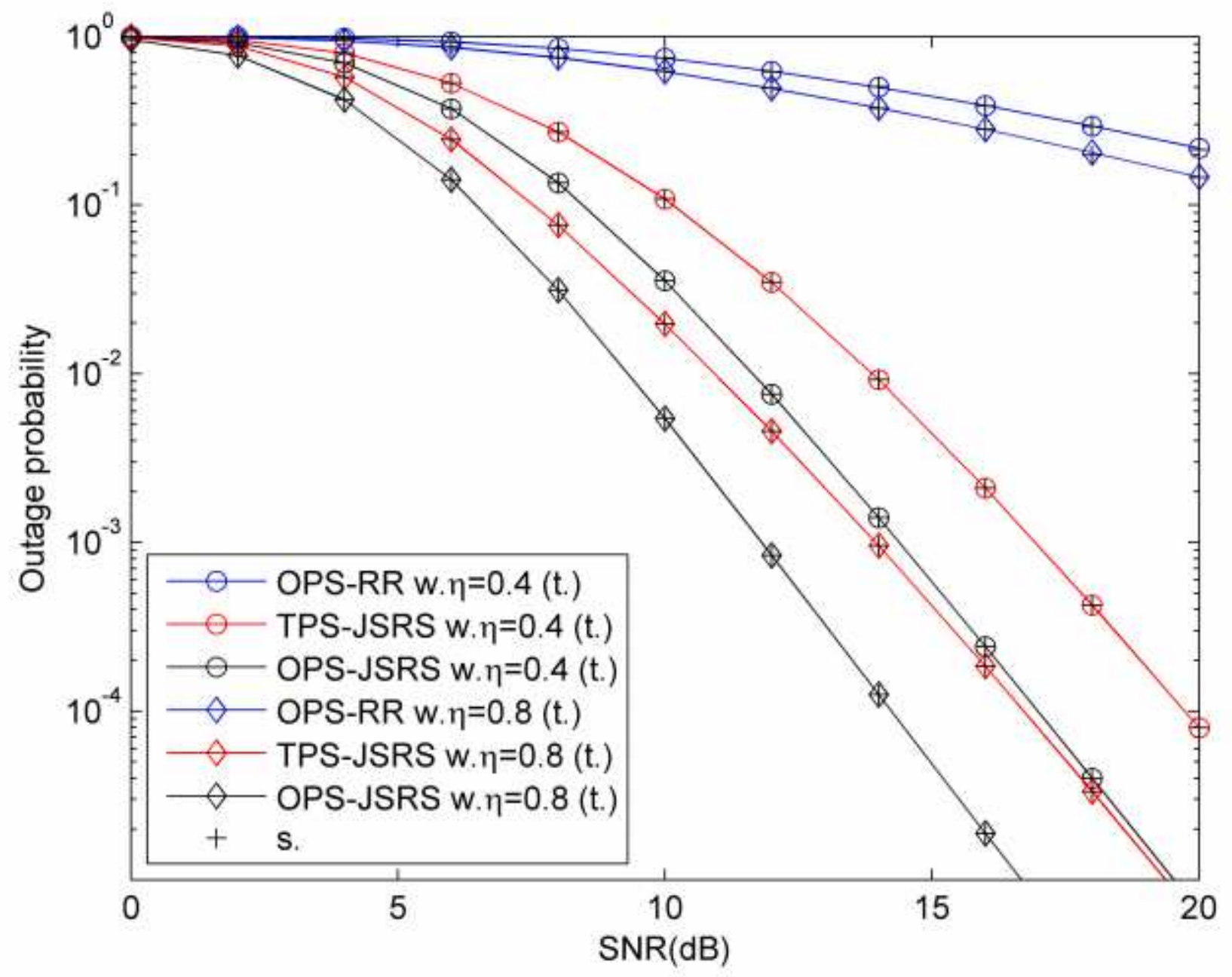

Figure 7

Please see the Manuscript PDF file for the complete figure caption 


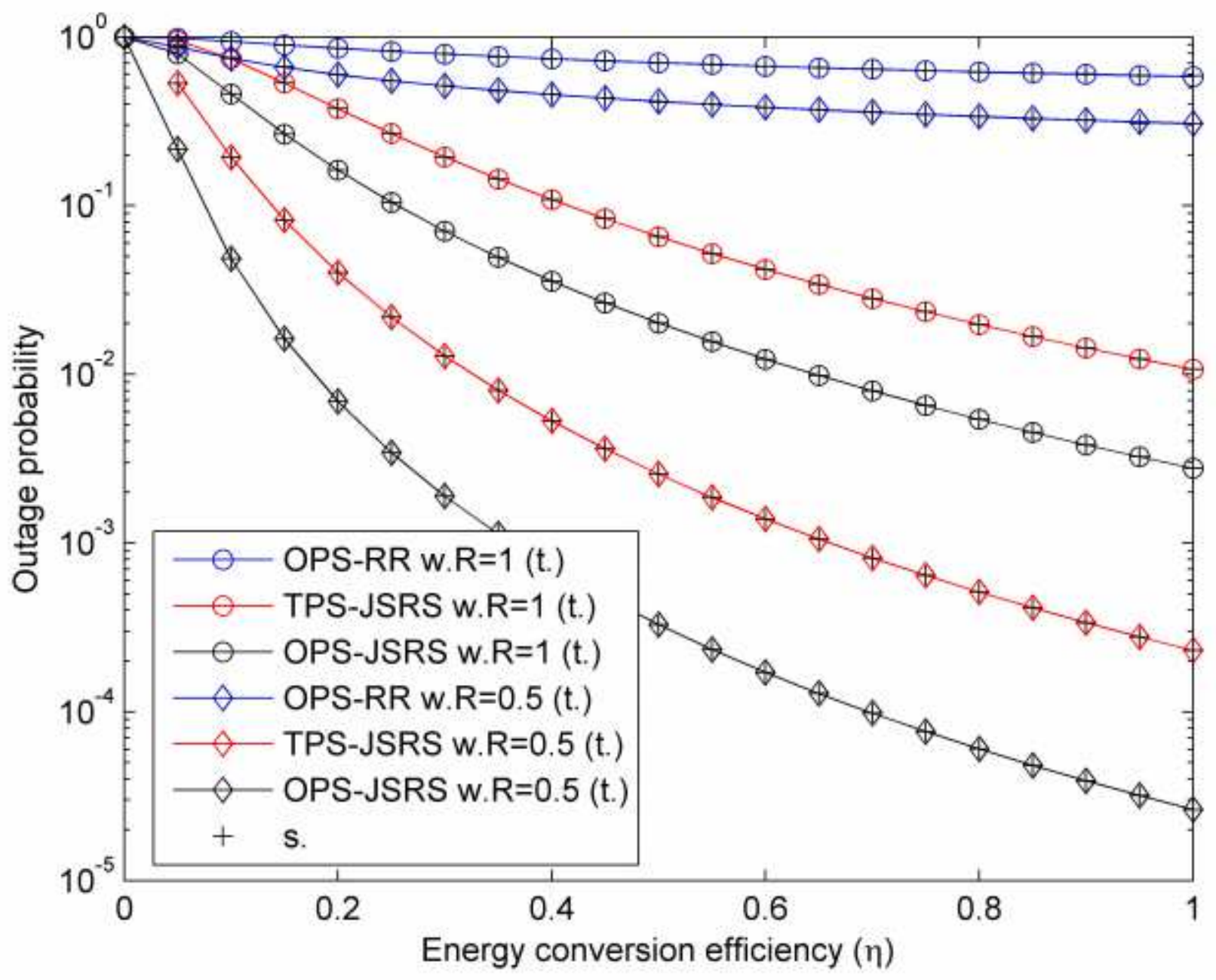

Figure 8

Please see the Manuscript PDF file for the complete figure caption 


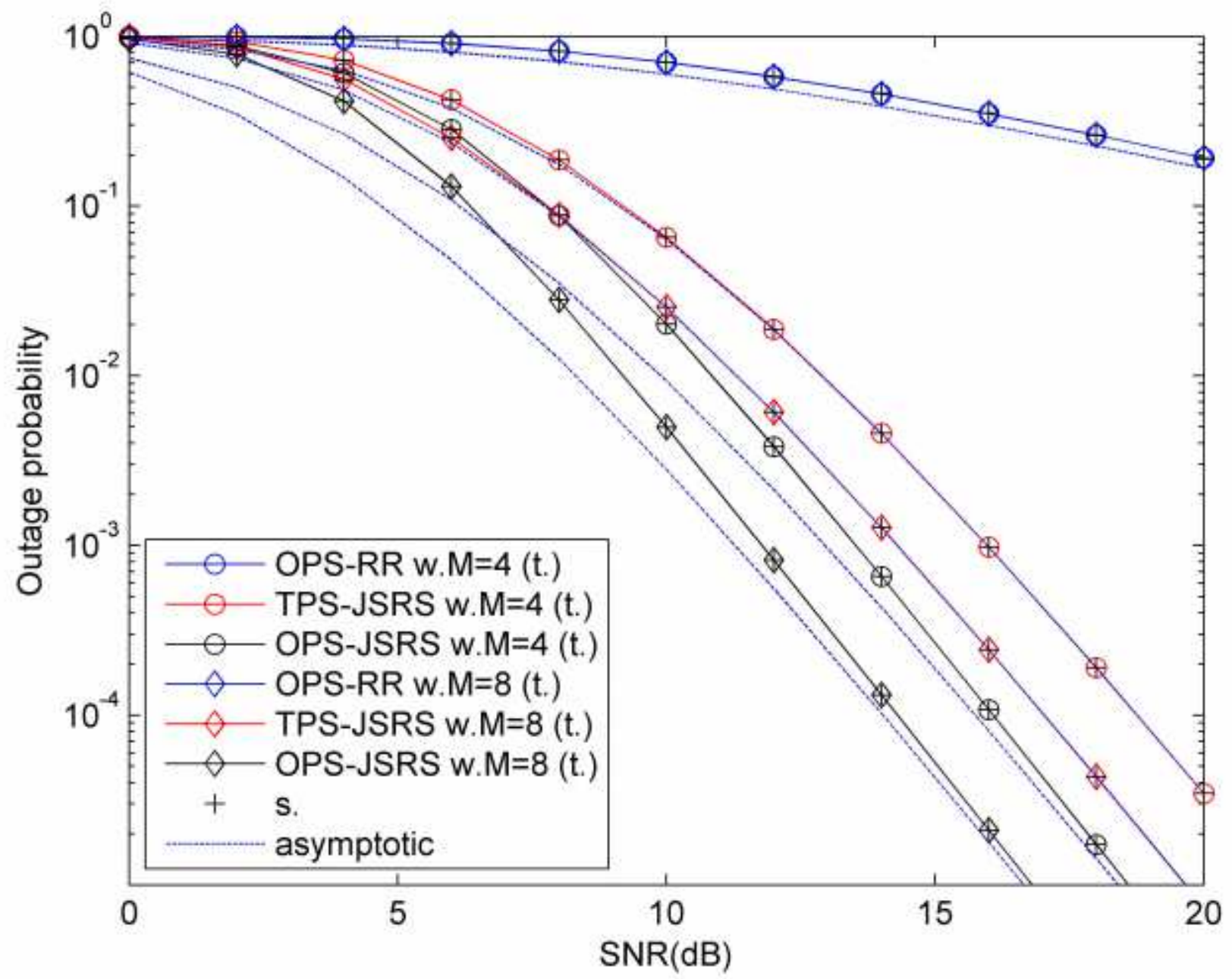

Figure 9

Please see the Manuscript PDF file for the complete figure caption 